\title{
Article \\ Evaluation of Nonsymmetric Macdonald Superpolynomials at Special Points
}

\author{
Charles F. Dunkl
}

check for

updates

Citation: Dunkl, C.F. Evaluation of Nonsymmetric Macdonald Superpolynomials at Special Points. Symmetry 2021, 13, 779. https:// doi.org/10.3390/sym13050779

Academic Editor: Alexei Kanel-Belov

Received: 12 April 2021

Accepted: 29 April 2021

Published: 1 May 2021

Publisher's Note: MDPI stays neutral with regard to jurisdictional claims in published maps and institutional affiliations.

Copyright: (C) 2021 by the author. Licensee MDPI, Basel, Switzerland. This article is an open access article distributed under the terms and conditions of the Creative Commons Attribution (CC BY) license (https:// creativecommons.org/licenses/by/ $4.0 /)$.
Department of Mathematics, University of Virginia, P.O. Box 400137, Charlottesville, VA 22904-4137, USA; cfd5z@virginia.edu

\begin{abstract}
In a preceding paper the theory of nonsymmetric Macdonald polynomials taking values in modules of the Hecke algebra of type A (Dunkl and Luque SLC 2012) was applied to such modules consisting of polynomials in anti-commuting variables, to define nonsymmetric Macdonald superpolynomials. These polynomials depend on two parameters $(q, t)$ and are defined by means of a Yang-Baxter graph. The present paper determines the values of a subclass of the polynomials at the special points $\left(1, t, t^{2}, \ldots\right)$ or $\left(1, t^{-1}, t^{-2}, \ldots\right)$. The arguments use induction on the degree and computations with products of generators of the Hecke algebra. The resulting formulas involve $(q, t)$-hook products. Evaluations are also found for Macdonald superpolynomials having restricted symmetry and antisymmetry properties.
\end{abstract}

Keywords: superpolynomials; Hecke algebra; hook products

MSC: 33D52; 20C08; 05E05

\section{Introduction}

In the prequel [1] of this paper we defined a representation of the Hecke algebra of type $A$ on spaces of superpolynomials. By using the theory of vector-valued nonsymmetric Macdonald polynomials developed by Luque and the author [2] we constructed nonsymmetric Macdonald superpolynomials. The basic theory including Cherednik operators, the Yang-Baxter graph method for computing the Macdonald superpolynomials, and norm formulas were described. The norm refers to an inner product with respect to which the generators of the Hecke algebra are self-adjoint. The theory relies on relating the Young tableaux approach to irreducible Hecke algebra modules to polynomials in anti-commuting variables. Furthermore, that paper showed how to produce symmetric and anti-symmetric Macdonald superpolynomials, and their norms, by use of the technique of Baker and Forrester [3]. In the present paper, we consider the evaluation of the polynomials at certain special points. The class of polynomials which lead to attractive formulas in pure product form is relatively small. These values are expressed by shifted $q$-factorials, both ordinary (positive integer labeled) and the type labeled by partitions, and $(q, t)$-hook products.

In Section 2, one finds the necessary background on the Hecke algebra of type $A$ and its representations on polynomials in anti-commuting (fermionic) variables and on superpolynomials which combine commuting (bosonic) and anti-commuting variables. This section also defines the Cherednik operators, a pairwise commuting set, whose simultaneous eigenvectors are called nonsymmetric Macdonald superpolynomials. They are constructed starting from degree zero by means of the Yang-Baxter graph. The necessary details from [1] are briefly given. Section 3 presents the main results with proofs about the evaluations; there are two types with similar arguments. The methods rely on steps in the graph to determine the values starting from degree zero. Some of the arguments are fairly technical computations using products of generators of the Hecke algebra. The definition of $(q, t)$-hook products and their use in the evaluation formulas are presented in Section 4. 
The evaluations are extended to Macdonald polynomials, of the types studied in the previous sections, with restricted symmetry and antisymmetry properties in Section 4 . The conclusion and ideas for further investigations in Section 6 conclude the paper.

\section{Background}

\subsection{The Hecke Algebra}

The Hecke algebra $\mathcal{H}_{N}(t)$ of type $A_{N-1}$ with parameter $t$ is the associative algebra over an extension field of $\mathbb{Q}$, generated by $\left\{T_{1}, \ldots, T_{N-1}\right\}$ subject to the braid relations

$$
\begin{gathered}
T_{i} T_{i+1} T_{i}=T_{i+1} T_{i} T_{i+1}, 1 \leq i<N-1, \\
T_{i} T_{j}=T_{j} T_{i},|i-j| \geq 2,
\end{gathered}
$$

and the quadratic relations

$$
\left(T_{i}-t\right)\left(T_{i}+1\right)=0,1 \leq i<N,
$$

where $t$ is a generic parameter (this means $t^{n} \neq 1$ for $2 \leq n \leq N$, and $t \neq 0$ ). The quadratic relation implies $T_{i}^{-1}=\frac{1}{t}\left(T_{i}+1-t\right)$. There is a commutative set of Jucys-Murphy elements in $\mathcal{H}_{N}(t)$ defined by $\omega_{N}=1, \omega_{i}=t^{-1} T_{i} \omega_{i+1} T_{i}$ for $1 \leq i<N$, that is,

$$
\omega_{i}=t^{i-N} T_{i} T_{i+1} \cdots T_{N-1} T_{N-1} T_{N-2} \cdots T_{i}
$$

Simultaneous eigenvectors of $\left\{\omega_{i}\right\}$ form bases of irreducible representations of the algebra. The symmetric group $\mathcal{S}_{N}$ is the group of permutations of $\{1,2, \ldots, N\}$ and is generated by the simple reflections (adjacent transpositions) $\left\{s_{i}: 1 \leq i<N\right\}$, where $s_{i}$ interchanges $i, i+1$ and fixes the other points (the $s_{i}$ satisfy the braid relations and $s_{i}^{2}=1$ ).

\subsection{Fermionic Polynomials}

Consider polynomials in $N$ anti-commuting (fermionic) variables $\theta_{1}, \theta_{2}, \ldots, \theta_{N}$. They satisfy $\theta_{i}^{2}=0$ and $\theta_{i} \theta_{j}+\theta_{j} \theta_{i}=0$ for $i \neq j$. The basis for these polynomials consists of monomials labeled by subsets of $\{1,2, \ldots, N\}$ :

$$
\phi_{E}:=\theta_{i_{1}} \cdots \theta_{i_{m}}, E=\left\{i_{1}, i_{2}, \cdots, i_{m}\right\}, 1 \leq i_{1}<i_{2}<\cdots<i_{m} \leq N .
$$

The polynomials have coefficients in an extension field of $\mathbb{Q}(q, t)$ with transcendental $q, t$, or generic $q, t$ satisfying $q, t \neq 0 q^{a} \neq 1, q^{a} t^{n} \neq 1$ for $a \in \mathbb{Z}$ and $n \neq 2,3, \ldots, N$.

Definition 1. $\mathcal{P}:=\operatorname{span}\left\{\phi_{E}: E \subset\{1, \ldots, N\}\right\}$ and $\mathcal{P}_{m}:=\operatorname{span}\left\{\phi_{E}: \# E=m\right\}$ for $0 \leq m \leq$ $N$. The fermionic degree of $\phi_{E}$ is \#E.

This is a brief description of the action of $T_{i}$ on $\mathcal{P}$ : suppose $j \in E_{1}$ implies $j<i$, and $j \in E_{2}$ implies $j>i+1$ : then

$$
\begin{aligned}
T_{i} \phi_{E_{1}} \theta_{i} \theta_{i+1} \phi_{E_{2}} & =-\phi_{E_{1}} \theta_{i} \theta_{i+1} \phi_{E_{2}}, T_{i} \phi_{E_{1}} \phi_{E_{2}}=t \phi_{E_{1}} \phi_{E_{2}} \\
T_{i} \phi_{E_{1}} \theta_{i} \phi_{E_{2}} & =\phi_{E_{1}} \theta_{i+1} \phi_{E_{2}}, T_{i} \phi_{E_{1}} \theta_{i+1} \phi_{E_{2}}=T_{i} \phi_{E_{1}}\left(t \theta_{i}+(t-1) \theta_{i+1}\right) \phi_{E_{2}} .
\end{aligned}
$$

Then $\left\{T_{i}: 1 \leq i<N\right\}$ satisfy the braid and quadratic relations.

There are two degree-changing linear maps which commute with the Hecke algebra action.

Definition 2. $\left\{\left\{\right.\right.$ For $n \in \mathbb{Z}$ set $\sigma(n):=(-1)^{n}$ and $\left.\}\right\}$ for $E \subset\{1,2, \ldots, N\}, 1 \leq i \leq N$ set $s(i, E):=\#\{j \in E: j<i\}$. Define the operators $\partial_{i}$ and $\widehat{\theta}_{i}$ by $\partial_{i} \theta_{i} \phi_{E}=\phi_{E}, \partial_{i} \phi_{E}=0$ and $\widehat{\theta}_{i} \phi_{E}=\theta_{i} \phi_{E}=(-1)^{s(i, E)} \phi_{E \cup\{i\}}$ for $i \notin E$, while $\widehat{\theta}_{i} \phi_{E}=0$ for $i \in E$ (also $i \in E$ implies $\phi_{E}=$ $(-1)^{s(i, E)} \theta_{i} \phi_{E \backslash\{i\}}$ and $\left.\partial_{i} \phi_{E}=(-1)^{s(i, E)} \phi_{E \backslash\{i\}}\right)$. Define $M:=\sum_{i=1}^{N} \widehat{\theta}_{i}$ and $D:=\sum_{i=1}^{N} t^{i-1} \partial_{i}$. 
It is clear that $D^{2}=0=M^{2}$. For $n=0,1,2, \ldots$ let $[n]_{t}:=\frac{1-t^{n}}{1-t}$.

Proposition 1. $M$ and $D$ commute with $T_{i}$ for $1 \leq i<N$, and $M D+D M=[N]_{t}$.

The spaces $\mathcal{P}_{m, 0}:=\operatorname{ker} D \cap \mathcal{P}_{m}$ and $\mathcal{P}_{m+1,1}:=\operatorname{ker} M \cap \mathcal{P}_{m+1}$ are irreducible $\mathcal{H}_{N}(t)$ modules and are isomorphic under the map $D: \mathcal{P}_{m+1,1} \rightarrow \mathcal{P}_{m, 0}$ and are of isotype $\left(N-m, 1^{m}\right)$.The representations of $\mathcal{H}_{N}(t)$ occurring in this paper correspond to reverse standard Young tableaus (RSYT) of hook shape (see Dipper and James [4] for details of the representation theory) These are labeled by partitions $\left(N-n, 1^{n}\right)$ of $N$ and are graphically described by Ferrers diagrams: boxes at $\{[1, i]: 1 \leq i \leq N-n\} \cup\{[j, 1]: 2 \leq j \leq n\}$. The numbers $\{1,2, \ldots, N\}$ are entered in the boxes in decreasing order in the row and in the column. For a given RSYT $Y$ let $Y[a, b]$ be the entry at $[a, b]$ and define the content $c(Y[a, b], Y):=b-a$. The vector $[c(i, Y): 1 \leq i \leq N]$ is called the content vector of $Y$. It defines $Y$ uniquely (trivially true for hook tableaux). The representation of $\mathcal{H}_{N}(t)$ is defined on the span of the RSYT's of shape $\left(N-n, 1^{n}\right)$ in such a way that $\omega_{i} Y=t^{c(i, Y)} Y$ for $1 \leq i \leq N$. We use a space-saving way of displaying an RSYT in two rows, with the second row consisting of the entries $Y_{E}[2,1], Y_{E}[3,1], \ldots$. Note that $Y[1,1]=N$ always.

As example let $N=8, n=3$,

$$
Y=\left[\begin{array}{lllll}
8 & 6 & 4 & 3 & 1 \\
\cdot & 7 & 5 & 2 &
\end{array}\right]
$$

and $[c(i, Y)]_{i=1}^{8}=[4,-3,3,2,-2,1,-1,0]$.

We showed [1] that $\mathcal{P}_{m}$ is a direct sum of the $\mathcal{H}_{N}(t)$-modules corresponding to $\left(N-m, 1^{m}\right)$ and $\left(N+1-m, 1^{m-1}\right) ; \operatorname{ker} D \cap \mathcal{P}_{m}$ and ker $M \cap \mathcal{P}_{m}$, respectively.

\subsection{The Module $\operatorname{ker} D \cap \mathcal{P}_{m}$}

The basis of $\operatorname{ker} D \cap \mathcal{P}_{m}$ is described as follows: Let $\mathcal{Y}_{0}:=\{E: \# E=m+1, N \in E\}$ and for $E \in \mathcal{Y}_{0}$ let $\psi_{E}=D \phi_{E}$. Associate $E$ to the RSYT $Y_{E}$ which contains the elements of $E$ in decreasing order in column 1 , that is, $\{[j, 1]: 1 \leq j \leq m+1\}$, and the elements of $E^{C}$ in $\{[1, i]: 2 \leq i \leq N-m\}$. In the example $Y=Y_{R}$ with $E=\{2,5,7,8\}$. The content vector of $E$ is defined by $c(i, E)=c\left(i, Y_{E}\right)$. For each $E \in \mathcal{Y}_{0}$ there is a polynomial $\tau_{E} \in$ $\operatorname{ker} D \cap \mathcal{P}_{m}$ such that $\omega_{i} \tau_{E}=t^{c(i, E)} \tau_{E}$ for $1 \leq i \leq N$, and if $\operatorname{inv}(E)=k$ then $\tau_{E}-t^{k} \psi_{E} \in$ $\operatorname{span}\left\{\psi_{E^{\prime}}: \operatorname{inv}\left(E^{\prime}\right)<k\right\}$. In particular if $E=\{N-m, N-m+1, \ldots, N\}$ then $\tau_{E}=D \phi_{E}$ (and this is one of the two cases that are used here). For example, suppose $N=7$, $m=3$ then

$$
Y_{E}=\left[\begin{array}{llll}
7 & 3 & 2 & 1 \\
\cdot & 6 & 5 & 4
\end{array}\right],
$$

$[c(i, E)]_{i=1}^{7}=[3,2,1,-3,-2,-1,0]$, and $\tau_{E}=D\left(\theta_{4} \theta_{5} \theta_{6} \theta_{7}\right)=t^{3} \theta_{5} \theta_{6} \theta_{7}-t^{4} \theta_{4} \theta_{6} \theta_{7}+$ $t^{5} \theta_{4} \theta_{5} \theta_{7}-t^{6} \theta_{4} \theta_{5} \theta_{6}$.

\subsection{The Module $\operatorname{ker} M \cap \mathcal{P}_{m}$}

The basis of $\operatorname{ker} M \cap \mathcal{P}_{m}$ is described as follows: Let $\mathcal{Y}_{1}:=\{F: \# F=m-1, N \notin F\}$ and for $F \in \mathcal{Y}_{1}$ let $\eta_{F}=M \phi_{F}$. Associate $F$ to the RSYT $Y_{F}$ which contains the elements of $F$ in decreasing order in column 1 , that is, $\{[j, 1]: 2 \leq j \leq m\}$, and the elements of $F^{C}$ in $\{[1, i]: 1 \leq i \leq N-m+11\}$. In the example (4) $Y=Y_{F}$ with $F=\{2,5,7\}$. As before the content vector of $F$ is defined by $c(i, F)=c\left(i, Y_{F}\right)$. For each $F \in \mathcal{Y}_{1}$ there is a polynomial $\tau_{F} \in \operatorname{ker} M \cap \mathcal{P}_{m}$ such that $\omega_{i} \tau_{F}=t^{c(i, F)} \tau_{F}$ for $1 \leq i \leq N$, and if inv $(F)=k$ then $\tau_{F}-\eta_{F} \in$ $\operatorname{span}\left\{\psi_{F^{\prime}}: \operatorname{inv}\left(F^{\prime}\right)>k\right\}$. Note that $F \in \mathcal{Y}_{1}$ implies $0 \leq \operatorname{inv}(F) \leq(m-1)(N-m+1)$ and the maximum value occurs at $F=\{1,2, \ldots, m-1\}$. This case is the second of those to be studied here. For this set $\tau_{F}=M \phi_{F}$. As example let $N=7, m=5$ then

$$
Y_{F}=\left[\begin{array}{lllll}
7 & 6 & 5 & & \\
\cdot & 4 & 3 & 2 & 1
\end{array}\right] \text {, }
$$




$$
[c(i, F)]_{i=1}^{7}=[-4,-3,-2,-1,2,1,0], \text { and } \tau_{F}=\theta_{1} \theta_{2} \theta_{3} \theta_{4}\left(\theta_{5}+\theta_{6}+\theta_{7}\right) .
$$

\subsection{Superpolynomials}

We extend the polynomials in $\left\{\theta_{i}\right\}$ by adjoining $N$ commuting variables $x_{1}, \ldots, x_{N}$ (that is $\left[x_{i}, x_{j}\right]=0,\left[x_{i}, \theta_{j}\right]=0, \theta_{i} \theta_{j}=-\theta_{j} \theta_{i}$ for all $i, j$ ). Each polynomial is a sum of monomials $x^{\alpha} \phi_{E}$ where $E \subset\{1,2, \ldots, N\}$ and $\alpha \in \mathbb{N}_{0}^{N}, x^{\alpha}:=\prod_{i=1}^{N} x_{i}^{\alpha_{i}}$. The partitions in $\mathbb{N}_{0}^{N}$ are denoted by $\mathbb{N}_{0}^{N,+}\left(\lambda \in \mathbb{N}_{0}^{N,+}\right.$ if and only if $\left.\lambda_{1} \geq \lambda_{2} \geq \ldots \geq \lambda_{N}\right)$. The fermionic degree of this monomial is \#E and the bosonic degree is $|\alpha|:=\sum_{i=1}^{N} \alpha_{i}$. The symmetric group $\mathcal{S}_{N}$ acts on the variables by $(x w)_{i}=x_{w(i)}$ and on exponents by $(w \alpha)_{i}=\alpha_{w^{-1}(i)}$ for $1 \leq i \leq N, w \in \mathcal{S}_{N}$ (consider $x$ as a row vector, $\alpha$ as a column vector and $w$ as a permutation matrix, $\widetilde{w}_{i, j}=\delta_{i, w(j)}$, then $x w=x \widetilde{w}$ and $\left.w \alpha=\widetilde{w} \alpha\right)$. Thus, $(x w)^{\alpha}=x^{w \alpha}$. Let $s \mathcal{P}_{m}:=$ $\operatorname{span}\left\{x^{\alpha} \phi_{E}: \alpha \in \mathbb{N}_{0}^{N}, \# E=m\right\}$. Then using the decomposition $\mathcal{P}_{m}=\mathcal{P}_{m, 0} \oplus \mathcal{P}_{m, 1}$ let

$$
\begin{aligned}
& s \mathcal{P}_{m, 0}=\operatorname{span}\left\{x^{\alpha} \psi_{E}: \alpha \in \mathbb{N}_{0}^{N}, E \in \mathcal{Y}_{0}\right\}, \\
& s \mathcal{P}_{m, 1}=\operatorname{span}\left\{x^{\alpha} \eta_{E}: \alpha \in \mathbb{N}_{0}^{N}, E \in \mathcal{Y}_{1}\right\} .
\end{aligned}
$$

The Hecke algebra $\mathcal{H}_{N}(t)$ is represented on $s \mathcal{P}_{m}$. This allows us to apply the theory of nonsymmetric Macdonald polynomials taking values in $\mathcal{H}_{N}(t)$-modules (see [2]).

Definition 3. Suppose $p \in s \mathcal{P}_{m}$ and $1 \leq i<N$ then set

$$
\boldsymbol{T}_{i} p(x ; \theta):=(1-t) x_{i+1} \frac{p(x ; \theta)-p\left(x s_{i} ; \theta\right)}{x_{i}-x_{i+1}}+T_{i} p\left(x s_{i} ; \theta\right) .
$$

Note that $T_{i}$ acts on the $\theta$ variables according to Formula (3).

Definition 4. Let $T^{(N)}=T_{N-1} T_{N-2} \cdots T_{1}$ and for $p \in s \mathcal{P}_{m}$ and $1 \leq i \leq N$

$$
\begin{aligned}
& w p(x ; \theta):=T^{(N)} p\left(q x_{N}, x_{1}, x_{2}, \ldots, x_{N-1} ; \theta\right), \\
& \xi_{i} p(x ; \theta):=t^{i-N} \boldsymbol{T}_{i} \boldsymbol{T}_{i+1} \cdots \boldsymbol{T}_{N-1} \boldsymbol{w} \boldsymbol{T}_{1}^{-1} \boldsymbol{T}_{2}^{-1} \cdots \boldsymbol{T}_{i-1}^{-1} p(x ; \theta) .
\end{aligned}
$$

The operators $\xi_{i}$ are Cherednik operators, defined by Baker and Forrester [5] (see Braverman et al. [6] for the significance of these operators in double affine Hecke algebras). They mutually commute (the proof in the vector-valued situation is in [2] [Thm. 3.8]). The simultaneous eigenfunctions are called nonsymmetric Macdonald polynomials. They have a triangularity property with respect to the partial order $\triangleright$ on the compositions $\mathbb{N}_{0}^{N}$, which is derived from the dominance order:

$$
\begin{gathered}
\alpha \prec \beta \Longleftrightarrow \sum_{j=1}^{i} \alpha_{j} \leq \sum_{j=1}^{i} \beta_{j}, 1 \leq i \leq N, \alpha \neq \beta, \\
\alpha \triangleleft \beta \Longleftrightarrow(|\alpha|=|\beta|) \wedge\left[\left(\alpha^{+} \prec \beta^{+}\right) \vee\left(\alpha^{+}=\beta^{+} \wedge \alpha \prec \beta\right)\right] .
\end{gathered}
$$

The rank function on compositions is involved in the formula for an NSMP.

Definition 5. For $\alpha \in \mathbb{N}_{0}^{N}, 1 \leq i \leq N$

$$
r_{\alpha}(i):=\#\left\{j: \alpha_{j}>\alpha_{i}\right\}+\#\left\{j: 1 \leq j \leq i, \alpha_{j}=\alpha_{i}\right\},
$$

then $r_{\alpha} \in \mathcal{S}_{N}$. There is a shortest expression $r_{\alpha}=s_{i_{1}} s_{i_{2}} \ldots s_{i_{k}}$ and $R_{\alpha}:=\left(T_{i_{1}} T_{i_{2}} \cdots T_{i_{k}}\right)^{-1} \in$ $\mathcal{H}_{N}(t)$ (that is, $R_{\alpha}=T\left(r_{\alpha}\right)^{-1}$ ). 
A consequence is that $r_{\alpha} \alpha=\alpha^{+}$, the nonincreasing rearrangement of $\alpha$, for any $\alpha \in \mathbb{N}_{0}^{N}$, and $r_{\alpha}=I$ if and only if $\alpha \in \mathbb{N}_{0}^{N,+}$.

Theorem 1 ([2] (Thm. 4.12)). Suppose $\alpha \in \mathbb{N}_{0}^{N}$ and $E \in \mathcal{Y}_{k}, k=0,1$ then there exists a $\left(\xi_{i}\right)$-simultaneous eigenfunction

$$
M_{\alpha, E}(x ; \theta)=t^{e\left(\alpha^{+}, E\right)} q^{\beta(\alpha)} x^{\alpha} R_{\alpha}\left(\tau_{E}(\theta)\right)+\sum_{\beta \triangleleft \alpha} x^{\beta} v_{\alpha, \beta, E}(\theta ; q, t)
$$

where $v_{\alpha, \beta, E}(\theta ; q, t) \in \mathcal{P}_{m, k}$ and its coefficients are rational functions of $q, t$. Furthermore, $\xi_{i} M_{\alpha, E}(x ; \theta)=\zeta_{\alpha, E}(i) M_{\alpha, E}(x ; \theta)$ where $\zeta_{\alpha, E}(i)=q^{\alpha_{i}} c^{c\left(r_{\alpha}(i), E\right)}$ for $1 \leq i \leq N$. The exponents $\beta(\alpha):=\sum_{i=1}^{N}\left(\begin{array}{c}\alpha_{i} \\ 2\end{array}\right)$ and $e\left(\alpha^{+}, E\right):=\sum_{i=1}^{N} \alpha_{i}^{+}(N-i+c(i, E))$.

The applications in the present paper require formulas for the transformation (called a step) $M_{\alpha, E} \rightarrow M_{s_{i} \alpha, E}$ when $\alpha_{i+1}>\alpha_{i}$ :

$$
M_{s_{i} \alpha, E}(x ; \theta)=\left(\boldsymbol{T}_{i}+\frac{1-t}{1-\zeta_{\alpha, E}(i+1) / \zeta_{\alpha, E}(i)}\right) M_{\alpha, E}(x ; \theta) .
$$

and for the affine step:

$$
\begin{aligned}
\Phi \alpha & =\left(\alpha_{2}, \alpha_{3}, \ldots, \alpha_{N}, \alpha_{1}+1\right) \\
\zeta_{\Phi \alpha, E} & =\left[\zeta_{\alpha, E}(2), \zeta_{\alpha, E}(3), \ldots, \zeta_{\alpha, E}(N), q \zeta_{\alpha, E}(1)\right] \\
M_{\Phi \alpha, E}(x) & =x_{N} w M_{\alpha, E}(x) .
\end{aligned}
$$

Two other key relations are $\zeta_{\alpha, E}(i+1)=t \zeta_{\alpha, E}(i)$ implies $\left(\boldsymbol{T}_{i}+1\right) M_{\alpha, E}=0$ and $\zeta_{\alpha, E}(i+1)=t^{-1} \zeta_{\alpha, E}(i)$ implies $\left(\boldsymbol{T}_{i}-t\right) M_{\alpha, E}=0$.

\section{Evaluations and Steps}

We consider two types of evaluations: (0) $x^{(0)}=\left(1, t, t^{2}, \ldots, t^{N-1}\right)$, $E=\{N-m, N-m+1, \ldots, N\}, \alpha \in \mathbb{N}_{0}^{N}$ with $\alpha_{i}=0$ for $N-m \leq i \leq N$, and $M_{\alpha, E}\left(x^{(0)}\right)=$ $V^{(0)}(\alpha) \tau_{E}$; (1) $x^{(1)}=\left(1, t^{-1}, t^{-2}, \ldots, t^{1-N}\right), F=\{1,2, \ldots, m\}, \alpha \in \mathbb{N}_{0}^{N}$ with $\alpha_{i}=0$ for $m+1 \leq i \leq N$, and $M_{\alpha, F}\left(x^{(1)}\right)=V^{(1)}(\alpha) \tau_{F}$.

Definition 6. Let $\mathcal{N}_{0}:=\left\{\alpha \in \mathbb{N}_{0}^{N}: i \geq N-m \Longrightarrow \alpha_{i}=0\right\}, \mathcal{N}_{0}^{+}:=\mathcal{N}_{0} \cap \mathbb{N}_{0}^{N,+}$. Let $\mathcal{N}_{1}:=$ $\left\{\alpha \in \mathbb{N}_{0}^{N}: i>m \Longrightarrow \alpha_{i}=0\right\}, \mathcal{N}_{1}^{+}:=\mathcal{N}_{1} \cap \mathbb{N}_{0}^{N,+}$.

Conceptually the two derivations are very much alike, but there are differences involving signs and powers of $t$ that need careful attention. We begin by expressing $V^{(0)}(\alpha)$ and $V^{(1)}(\alpha)$ in terms of $V^{(0)}\left(\alpha^{+}\right)$and $V^{(1)}\left(\alpha^{+}\right)$. Since we are concerned with evaluations the following is used throughout:

Definition 7. For a fixed point $x \in \mathbb{R}^{N}$ and $1 \leq i<N$ let $b(x ; i)=\frac{t-1}{1-x_{i} / x_{i+1}}\left(x_{i} \neq x_{i+1}\right)$. In particular if $x_{i+1}=t^{n} x_{i}$ then let $\kappa_{n}:=b(x ; i)$ for $n \in \mathbb{Z} \backslash\{0\}$. If $n \geq 1$ then $\kappa_{n}=\frac{t^{n}}{[n]_{t}}$ and $\kappa_{-n}=-\frac{1}{[n]_{t}}$.

In terms of $b$ the evaluation formula for $\boldsymbol{T}_{i}$ is

$$
\boldsymbol{T}_{i} p(x ; \theta)=b(x ; i) p(x ; \theta)+\left(T_{i}-b(x ; i)\right) p\left(x s_{i} ; \theta\right)
$$

The following are used repeatedly in the sequel. 
Lemma 1. Suppose for some $i<N$ there is a polynomial $p(x ; \theta)$ and a point $y$ such that $\left(\boldsymbol{T}_{i}+1\right) p=0$ and $y_{i+1}=$ ty $y_{i}$ then $\left(T_{i}+1\right) p(y ; \theta)=0$.

Proof. By hypothesis $b(y ; i)=t$ and thus $(1+t) p(y ; \theta)+\left(T_{i}-t\right) p\left(y s_{i} ; \theta\right)=0$. Then $(1+t)\left(T_{i}+1\right) p(y ; \theta)=-\left(T_{i}+1\right)\left(T_{i}-t\right) p\left(y s_{i} ; \theta\right)=0$.

Lemma 2. Suppose for some $i<N$ there is a polynomial $p(x ; \theta)$ and a point $y$ such that $\left(\boldsymbol{T}_{i}-t\right) p=0$ and $y_{i}=t y_{i+1}$ then $\left(T_{i}-t\right) p(y ; \theta)=0$.

Proof. By hypothesis $b(y ; i)=-1$ and thus $(-t-1) p(y ; \theta)+\left(T_{i}+1\right) p\left(y s_{i} ; \theta\right)=0$. Then $(1+t)\left(T_{i}-t\right) p(y ; \theta)=-\left(T_{i}-t\right)\left(T_{i}+1\right) p\left(y s_{i} ; \theta\right)=0$.

In type $(0) \zeta_{\alpha, E}(i)=t^{i-N}$ for $N-m \leq i \leq N$ which implies $\left(\boldsymbol{T}_{i}+1\right) M_{\alpha, E}=0$ for $N-m \leq i<N$.

Lemma 3. Suppose $M_{\alpha, E}$ is of type (0) and $x_{i+1}=t x_{i}$ for $N-m \leq i<N$ then $M_{\alpha, E}(x)=c \tau_{E}$ for some constant depending on $x$, and $\left(T_{i}-t\right) M_{\alpha, E}(x)=0$ for $1 \leq i<N-m-1$.

Proof. From $\left(\boldsymbol{T}_{i}+1\right) M_{\alpha, E}=0$ and Lemma 1 it follows that $\left(T_{i}+1\right) M_{\alpha, E}(x)=0$ for $N-m \leq i<N$. Thus, $\left(\omega_{i}-t^{i-N}\right) M_{\alpha, E}(x ; \theta)=0$ for $N-m \leq i \leq N$, and this implies $M_{\alpha, E}(x ; \theta)$ is a multiple of $\tau_{E}$ (the contents $\left[c\left(i, E^{\prime}\right)\right]_{i=N-m}^{N}$ determine $E^{\prime}$ uniquely). Furthermore $\left(T_{i}-t\right) \tau_{E}=0$ for $1 \leq i<N-m-1$ (since $1,2, \ldots, N-m-1$ are in the same row of $\left.Y_{E}\right)$.

Proposition 2. Suppose $\alpha \in \mathcal{N}_{0}$ and $\alpha_{i}<\alpha_{i+1}$ (implying $i+1<N-m$ ) and $z=\zeta_{\alpha, E}(i+1) / \zeta_{\alpha, E}(i)$ then

$$
M_{s_{i} \alpha, E}\left(x^{(0)} ; \theta\right)=\frac{1-t z}{1-z} M_{\alpha, E}\left(x^{(0)} ; \theta\right)
$$

Proof. From $(7)$ and $(8)$ with $b\left(x^{(0)}, i\right)=t$ it follows that

$$
\begin{aligned}
M_{s_{i} \alpha, E}\left(x^{(0)} ; \theta\right) & =\left(t+\frac{1-t}{1-z}\right) M_{\alpha, E}\left(x^{(0)} ; \theta\right)+\left(T_{i}-t\right) M_{\alpha, E}\left(x^{(0)} s_{i} ; \theta\right) \\
& =\frac{1-t z}{1-z} M_{\alpha, E}\left(x^{(0)} ; \theta\right)
\end{aligned}
$$

because $x^{(0)} s_{i}$ satisfies the hypotheses of the Lemma implying $\left(T_{i}-t\right) M_{\alpha, E}\left(x^{(0)} s_{i} ; \theta\right)=0$.

The following products are used to relate $V^{(k)}(\alpha)$ to $V^{(k)}\left(\alpha^{+}\right), k=0,1$.

Definition 8. Let $u_{0}(z):=\frac{t-z}{1-z}, u_{1}(z):=\frac{1-t z}{1-z}$. Suppose $\beta \in \mathbb{N}_{0}^{N}$ and $E^{\prime} \in \mathcal{Y}^{0} \cup \mathcal{Y}^{1}$ and $k=0,1$ then

$$
\mathcal{R}_{k}\left(\beta, E^{\prime}\right):=\prod_{1 \leq i<j \leq N, \beta_{i}<\beta_{j}} u_{k}\left(q^{\beta_{j}-\beta_{i}} t^{c\left(r_{\beta}(j), E^{\prime}\right)-c\left(r_{\beta}(i), E^{\prime}\right)}\right)
$$

Note that the argument of $u_{k}$ is $\zeta_{\beta, E^{\prime \prime}}(j) / \zeta_{\beta, E^{\prime \prime}}(i)$ and there are inv $(\beta)$ factors, where

$$
\operatorname{inv}(\beta):=\left\{(i, j): 1 \leq i<j \leq N, \beta_{i}<\beta_{j}\right\}
$$

Lemma 4. If $\beta_{i}<\beta_{i+1}$ then $\mathcal{R}_{k}\left(\beta, E^{\prime}\right)=u_{k}\left(\zeta_{\beta, E^{\prime \prime}}(i+1) / \zeta_{\beta, E^{\prime \prime}}(i)\right) \mathcal{R}_{k}\left(s_{i} \beta, E^{\prime}\right)$. 
Proof. The only factor that appears in $\mathcal{R}_{k}\left(\beta, E^{\prime}\right)$ but not in $\mathcal{R}_{k}\left(s_{i} \beta, E^{\prime}\right)$ is $u_{k}\left(\zeta_{\beta, E^{\prime \prime}}(i+1) / \zeta_{\beta, E^{\prime \prime}}(i)\right)$.

For the special case type $(0) 1 \leq r_{\alpha}(i)<N-m$ we find $c\left(r_{\alpha}(i), E\right)=N-m-r_{\alpha}(i)$ and

$$
\mathcal{R}_{1}(\alpha, E)=\prod_{1 \leq i<j<N-m, \alpha_{i}<\alpha_{j}} u_{1}\left(q^{\alpha_{j}-\alpha_{i}} t^{r_{\alpha}(i)-r_{\alpha}(j)}\right) .
$$

Proposition 3. Suppose $\alpha \in \mathcal{N}_{0}$ then $M_{\alpha, E}\left(x^{(0)} ; \theta\right)=V^{(0)}(\alpha) \tau_{E}$ and

$$
V^{(0)}(\alpha)=\mathcal{R}_{1}(\alpha, E)^{-1} V^{(0)}\left(\alpha^{+}\right) .
$$

Proof. By Lemma $3 M_{\alpha, E}\left(x^{(0)} ; \theta\right)$ is a multiple of $\tau_{E}$. For the product formula argue by induction on $\operatorname{inv}(\alpha)$. If $\lambda \in \mathbb{N}_{0}^{N,+}$ then $\mathcal{R}_{1}(\lambda, E)=1$. If $\alpha_{i}<\alpha_{i+1}$ then

$$
\frac{V^{(0)}\left(s_{i} \alpha\right)}{V^{(0)}(\alpha)}=u_{1}\left(\frac{\zeta_{\alpha, E}(i+1)}{\zeta_{\alpha, E}(i)}\right)=\frac{\mathcal{R}_{1}(\alpha, E)}{\mathcal{R}_{1}\left(s_{i} \alpha, E\right)} .
$$

In type (1) $\zeta_{\alpha}(i)=t^{N-i}$ for $m+1 \leq i \leq N$ which implies $\left(\boldsymbol{T}_{i}-t\right) M_{\alpha, E}=0$ for $m+1 \leq i<N$.

Lemma 5. Suppose $M_{\alpha, F}$ is of type (1) and $x_{i}=t x_{i+1}$ for $m+1 \leq i<N$ then $M_{\alpha, E}(x)=c \tau_{F}$ for some constant depending on $x$, and $\left(T_{i}+1\right) M_{\alpha, F}(x)=0$ for $1 \leq i<m$.

Proof. From $\left(\boldsymbol{T}_{i}-t\right) M_{\alpha, F}=0$ and Lemma 2 it follows that $\left(T_{i}-t\right) M_{\alpha, F}(x ; \theta)=0$ for $m+1 \leq i<N$. Thus, $\left(\omega_{i}-t^{N-i}\right) M_{\alpha, F}(x ; \theta)=0$ for $m+1 \leq i \leq N$, and this implies $M_{\alpha, F}(x ; \theta)$ is a multiple of $\tau_{F}$ (the contents $\left[c\left(i, E^{\prime}\right)\right]_{i=m+1}^{N}$ determine $E^{\prime}$ uniquely). Thus, $\left(T_{i}+1\right) \tau_{F}=0$ for $1 \leq i<m$ (since $1,2, \ldots, m$ are in the same column of $Y_{F}$ ).

Proposition 4. Suppose $\alpha \in \mathcal{N}_{1}$ and $\alpha_{i}<\alpha_{i+1}$ (so that $i+1 \leq m$ ) and $z=\zeta_{\alpha, F}(i+1) / \zeta_{\alpha, F}(i)$ then

$$
M_{s_{i} \alpha, F}\left(x^{(1)} ; \theta\right)=-\frac{t-z}{1-z} M_{\alpha, F}\left(x^{(1)} ; \theta\right) .
$$

Proof. From (7) and (8) with $b\left(x^{(1)}, i\right)=-1$ it follows that

$$
\begin{aligned}
M_{s_{i} \alpha, F}\left(x^{(1)} ; \theta\right) & =\left(-1+\frac{1-t}{1-z}\right) M_{\alpha, F}\left(x^{(1)} ; \theta\right)+\left(T_{i}+1\right) M_{\alpha, F}\left(x^{(1)} s_{i} ; \theta\right) \\
& =-\frac{t-z}{1-z} M_{\alpha, F}\left(x^{(1)} ; \theta\right),
\end{aligned}
$$

because $x^{(1)} s_{i}$ satisfies the hypotheses of the Lemma implying $\left(T_{i}+1\right) M_{\alpha, F}\left(x^{(1)} s_{i} ; \theta\right)=0$.

Proposition 5. Suppose $\alpha \in \mathcal{N}_{1}$ then $M_{\alpha, F}\left(x^{(1)} ; \theta\right)=V^{(1)}(\alpha) \tau_{F}$ and

$$
V^{(1)}(\alpha)=(-1)^{\operatorname{inv}(\alpha)} \mathcal{R}_{0}(\alpha, F)^{-1} V^{(1)}\left(\alpha^{+}\right) .
$$


Proof. By Lemma $5 M_{\alpha, F}\left(x^{(1)} ; \theta\right)$ is a multiple of $\tau_{F}$. For the product formula argue by induction on $\operatorname{inv}(\alpha)$. If $\lambda \in \mathbb{N}_{0}^{N,+}$ then $\mathcal{R}_{0}(\lambda, F)=1$. If $\alpha_{i}<\alpha_{i+1}$ then

$$
\frac{V^{(1)}\left(s_{i} \alpha\right)}{V^{(1)}(\alpha)}=-u_{0}\left(\frac{\zeta_{\alpha, F}(i+1)}{\zeta_{\alpha, F}(i)}\right)=-\frac{\mathcal{R}_{0}(\alpha, F)}{\mathcal{R}_{0}\left(s_{i} \alpha, F\right)} .
$$

We will use induction on the last nonzero part of $\lambda \in \mathbb{N}_{0}^{N,+}$ to derive $V^{(*)}(\lambda)$. Suppose $\lambda_{k} \geq 1$ and $\lambda_{i}=0$ for $i>k$ where $1 \leq k \leq N-m-1$ in type (0) and $1 \leq k \leq m$ in type (1). Define compositions in $\mathbb{N}_{0}^{N}$ by

$$
\begin{gathered}
\lambda^{\prime}=\left(\lambda_{1}, \ldots, \lambda_{k-1}, \lambda_{k}-1,0, \ldots\right) \\
\alpha=\left(\lambda_{k}-1, \lambda_{1}, \ldots, \lambda_{k-1}, 0, \ldots\right) \\
\beta=\left(\lambda_{1}, \ldots, \lambda_{k-1}, 0, \ldots, \lambda_{k}\right) \\
\delta=\left(\lambda_{1}, \ldots, \lambda_{k-1}, 0, \ldots, \lambda_{k}, \stackrel{n+1}{0}, 0 \ldots\right) \\
\lambda=\left(\lambda_{1}, \ldots, \lambda_{k-1}, \lambda_{k}, 0, \ldots\right),
\end{gathered}
$$

where $n=N-m-1$ in type (0) and $n=m$ in type (1). The transitions from $\lambda^{\prime} \rightarrow \alpha$ and from $\delta \rightarrow \lambda$ use Propositions 3 and 5. The affine step $\alpha \rightarrow \beta$ and the steps $\beta \rightarrow \delta$ require technical computations.

Proposition 6. Suppose $\lambda \in \mathcal{N}_{0}^{+}$and $\lambda^{\prime}, \alpha$ are given by (9) then

$$
V^{(0)}(\alpha)=V^{(0)}\left(\lambda^{\prime}\right) \prod_{i=1}^{k-1} \frac{1-q^{\lambda_{i}-\lambda_{k}+1} t^{k-i}}{1-q^{\lambda_{i}-\lambda_{k}+1} t^{k-i+1}} .
$$

Proof. The spectral vector of $\lambda^{\prime}$ has $\zeta_{\lambda^{\prime}, E}(i)=q^{\lambda_{i} t^{N-m-i}}$ for $1 \leq i<k, \zeta_{\lambda^{\prime}, E}(k)=$ $q^{\lambda_{k}-1} t^{N-m-k}$ while $\zeta_{\alpha, E}(1)=\zeta_{\lambda^{\prime}, E}(k)$ and $\zeta_{\alpha, E}(i)=\zeta_{\lambda^{\prime}, E}(i-1)$ for $2 \leq i \leq k$. The product is $\mathcal{R}_{1}(\alpha, E)^{-1}$.

Proposition 7. Suppose $\lambda \in \mathcal{N}_{0}^{+}$and $\delta$ is as in (10) then

$$
V^{(0)}(\lambda)=\frac{1-q^{\lambda_{k}} t^{N-m-k}}{1-q^{\lambda_{k} t}} V^{(0)}(\delta)
$$

Proof. The relevant part of $\zeta_{\delta, E}$ is $\zeta_{\delta, E}(i)=t^{N-m-i-1}$ for $k \leq i \leq N-m-2$ and $\zeta_{\delta, E}(N-m-1)=q^{\lambda} t^{N-m-k}$. Thus

$$
\mathcal{R}_{1}(\delta, E)=\prod_{i=k}^{N-m-2} u_{1}\left(q^{\lambda_{k}} t^{i+1-k}\right)=\prod_{j=0}^{N-m-k-2} \frac{1-q^{\lambda_{k}} t^{j+2}}{1-q^{\lambda_{k} t^{j+1}}}
$$

and this product telescopes.

Proposition 8. Suppose $\lambda \in \mathcal{N}_{1}^{+}$and $\lambda^{\prime}, \alpha$ are given by (9) then

$$
V^{(1)}(\alpha)=(-t)^{1-k} \prod_{i=1}^{k-1} \frac{1-q^{\lambda_{i}-\lambda_{k}+1} t^{i-k}}{1-q^{\lambda_{i}-\lambda_{k}+1} t^{i-k-1}} V^{(1)}\left(\lambda^{\prime}\right) .
$$


Proof. The spectral vector of $\lambda^{\prime}$ has $\zeta_{\lambda^{\prime}, F}(i)=q^{\lambda_{i}} t^{i-1-m}$ for $1 \leq i<m, \zeta_{\lambda^{\prime}, F}(k)=$ $q^{\lambda_{k}-1} t^{k-1-m}$ while $\zeta_{\alpha, F}(1)=\zeta_{\lambda^{\prime}, F}(k)$ and $\zeta_{\alpha, F}(i)=\zeta_{\lambda^{\prime}, F}(i-1)$ for $2 \leq i \leq k$. Furthermore, $\operatorname{inv}(\alpha)=k-1$. Then

$$
\mathcal{R}_{0}(\alpha, F)=\prod_{i=1}^{k-1} u_{0}\left(q^{\lambda_{i}-\lambda_{k}+1} t^{i-k}\right)=t^{k-1} \prod_{i=1}^{k-1} \frac{1-q^{\lambda_{i}-\lambda_{k}+1} t^{i-k-1}}{1-q^{\lambda_{i}-\lambda_{k}+1} t^{i-k}} .
$$

Combine this with $V^{(1)}(\alpha)=(-1)^{\operatorname{inv}(\alpha)} \mathcal{R}_{0}(\alpha, F)^{-1} V^{(1)}\left(\lambda^{\prime}\right)$.

Proposition 9. Suppose $\lambda \in \mathcal{N}_{1}^{+}$and $\delta$ is as in (10) then

$$
V^{(1)}(\lambda)=(-t)^{m-k} \frac{1-q^{\lambda_{k}} t^{k-m-1}}{1-q^{\lambda_{k} t^{-1}}} V^{(1)}(\delta) .
$$

Proof. The relevant part of $\zeta_{\delta, F}$ is $\zeta_{\delta, F}(i)=t^{i-m}$ for $k \leq i \leq m-1$ and $\zeta_{\delta, E}(m)=q^{\lambda_{k}} t^{k-m-1}$. Thus

$$
\mathcal{R}_{0}(\delta, E)=\prod_{i=k}^{m-1} u_{0}\left(q^{\lambda_{k}} t^{k-i-1}\right)=t^{m-k} \prod_{j=0}^{m-k-1} \frac{1-q^{\lambda_{k}} t^{-j-2}}{1-q^{\lambda_{k} t^{-j-1}}}
$$

and this product telescopes to $\frac{1-q^{\lambda_{k} t^{k-m-1}}}{1-q^{\lambda_{k} t^{-1}}}$. The use of $\operatorname{inv}(\delta)=m-k$ completes the proof.

The methods used in these calculations are similar to those used in [7] for evaluations of scalar valued Macdonald polynomials, however the following computations (from $\alpha$ to $\delta$ ) are significantly different.

Each of the remaining transitions is calculated in its own subsection. The following two lemmas will be used in both types. Recall $t \boldsymbol{T}_{i}^{-1}=1-t+\boldsymbol{T}_{i}$ for any $i$.

Lemma 6. Suppose $f=t \boldsymbol{T}_{i}^{-1} g$ and $b=b(x ; i)$ then

$$
\left(T_{i}-b\right) f\left(x s_{i}\right)=(1+b)(t-b) g(x)-b\left(T_{i}-b\right) g\left(x s_{i}\right)
$$

Proof. From $g=t^{-1} \boldsymbol{T}_{i} f$ we get

$$
\begin{aligned}
\operatorname{tg}(x) & =b f(x)+\left(T_{i}-b\right) f\left(x s_{i}\right) \\
f(x) & =(1-t+b) g(x)+\left(T_{i}-b\right) g\left(x s_{i}\right),
\end{aligned}
$$

thus

$$
\begin{aligned}
\left(T_{i}-b\right) f\left(x s_{i}\right) & =\operatorname{tg}(x)-b(1-t+b) g(x)-b\left(T_{i}-b\right) g\left(x s_{i}\right) \\
& =(1+b)(t-b) g(x)-b\left(T_{i}-b\right) g\left(x s_{i}\right) .
\end{aligned}
$$

The next formula is a modified braid relation.

Lemma 7. Suppose $b=\frac{a c}{a+c+1-t}$ or $j, \ell, j+\ell \in \mathbb{Z} \backslash\{0\}$ then

$$
\begin{aligned}
\left(T_{i}-a\right)\left(T_{i+1}-b\right)\left(T_{i}-c\right) & =\left(T_{i+1}-c\right)\left(T_{i}-b\right)\left(T_{i+1}-a\right) \\
\left(T_{i}-\kappa_{j}\right)\left(T_{i+1}-\kappa_{j+\ell}\right)\left(T_{i}-\kappa_{\ell}\right) & =\left(T_{i+1}-\kappa_{\ell}\right)\left(T_{i}-\kappa_{j+\ell}\right)\left(T_{i+1}-\kappa_{j}\right)
\end{aligned}
$$


Proof. Expand

$$
\begin{aligned}
& \left(T_{i}-a\right)\left(T_{i+1}-b\right)\left(T_{i}-c\right)+c T_{i} T_{i+1}+a T_{i+1} T_{i}+a b c \\
& =T_{i} T_{i+1} T_{i}+a c T_{i+1}+b(a+c) T_{i}-b T_{i}^{2} \\
& =T_{i} T_{i+1} T_{i}-b t+a c T_{i+1}+b(a+c-t+1) T_{i} \\
& =T_{i} T_{i+1} T_{i}-b t+a c\left(T_{i+1}+T_{i}\right) .
\end{aligned}
$$

which is symmetric in $T_{i}, T_{i+1}$ since $T_{i} T_{i+1} T_{i}=T_{i+1} T_{i} T_{i+1}$. If $a=\kappa_{j}$ and $c=\kappa_{\ell}$ then $b=\kappa_{j+\ell}$.

\subsection{From $\alpha$ to $\delta$ for Type (0)}

In this section, we will prove $M_{\delta, E}\left(x^{(0)}\right)=q^{\lambda_{k}-1} t^{2 N-m-k-1} \frac{1-q^{\lambda_{k}} t^{N-k+1}}{1-q^{\lambda_{k}} t^{N-k}} M_{\alpha, E}\left(x^{(0)}\right)$. Start with $\delta$ (where $\delta_{i}=\lambda_{i}$ for $1 \leq i \leq k-1, \delta_{N-m-1}=\lambda_{k}$ and $\delta_{i}=0$ otherwise). Let $\beta^{(N-m-1)}=\delta$ and $\beta^{(j)}=s_{j-1} \beta^{(j-1)}$ for $N-m \leq j \leq N$ (so that $\beta^{(N)}=\beta$ in (9)). Abbreviate

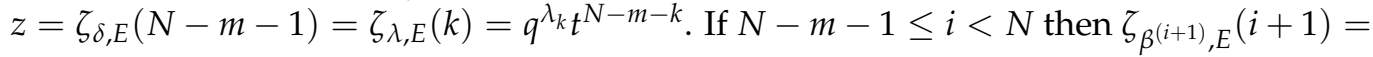
$z, \zeta_{\beta^{(i+1)}, E}(i)=t^{i+1-N}$. Set $p_{i}(x)=M_{\beta^{(i)}, E}(x ; \theta)$ for $N-m-1 \leq i \leq N$, then

$$
\begin{aligned}
p_{i}(x) & =\left(\boldsymbol{T}_{i-1}+\frac{1-t}{1-z t^{N-i-1}}\right) p_{i+1}(x) \\
& =\left(b(x ; i)+\frac{1-t}{1-z t^{N-i-1}}\right) p_{i+1}(x)+\left(T_{i}-b(x ; i)\right) p_{i+1}\left(x s_{i}\right) .
\end{aligned}
$$

To start set $i=N-m-1$ and $x=x^{(0)}$ (thus $\left.b\left(x^{(0)} ; i\right)=t\right)$

$$
p_{N-m-1}\left(x^{(0)}\right)=\frac{1-z t^{m+1}}{1-z t^{m}} p_{N-m}\left(x^{(0)}\right)+\left(T_{N-m-1}-t\right) p_{N-m}\left(x^{(0)} s_{N-m-1}\right) .
$$

Two series of points are used in the calculation: Define $y_{N-m-1}=x^{(0)}, y_{N-m}=$ $x^{(0)} s_{N-m}, y_{i}=y_{i-1} s_{i}$ for $N-m+1 \leq i \leq N-1$; define $v_{N-m-2}=x^{(0)}, v_{i}=v_{i-1} s_{i}$ for $N-m-1 \leq i \leq N-1$. Thus

$$
\begin{aligned}
& y_{i-1}=\left(\ldots, t^{N-m-2}, t^{N-m}, \ldots, t^{N-m-1}, t^{i}, \ldots, t^{N-1}\right), b\left(y_{i-1} ; i\right)=\kappa_{i+m-N+1}, \\
& v_{i-1}=\left(\ldots, t^{N-m-1}, t^{N-m}, \ldots, t^{N-m-2}, t^{i}, \ldots, t^{N-1}\right), b\left(v_{i-1} ; i\right)=\kappa_{i+m-N+2} .
\end{aligned}
$$

Lemma 8. Suppose $N-m \leq j<N$ and $\boldsymbol{T}_{j} p=-p$ then $\left(T_{j}-\kappa_{j-N+m+1}\right) p\left(y_{j}\right)$ $=-\frac{[j-N+m+2]_{t}}{[j-N+m+1]_{t}} p\left(y_{j-1}\right)$ and $\left(T_{j}-\kappa_{j-N+m+2}\right) p\left(v_{j}\right)=-\frac{[j-N+m+3]_{t}}{[j-N+m+2]_{t}} p\left(v_{j-1}\right)$ for $1 \leq j \leq i$.

Proof. This follows from

$$
\left(T_{j}-b(x ; j)\right) p\left(x s_{j}\right)=-(1+b(x ; j)) p(x)
$$

with $x=y_{j-1}$ so that $x s_{j}=y_{j}=y_{j-1}$, and with $x=v_{j-1}$. If $n=1,2, \ldots$ then $1+\kappa_{n}=$ $\frac{1+t+\cdots+t^{n-1}+t^{n}}{1+t+\cdots+t^{n-1}}=\frac{[n+1]_{t}}{[n]_{t}}$. 
Proposition 10. For $N-m \leq i \leq N$

$$
\begin{aligned}
p_{N-m-1}\left(x^{0}\right) & =\frac{1-z t^{m+1}}{1-z t^{m}}\left(T_{N-m}-t\right) \cdots\left(T_{i-1}-\kappa_{i-N+m}\right) p_{i}\left(y_{i-1}\right) \\
& +\left(T_{N-m-1}-t\right)\left(T_{N-m}-\kappa_{2}\right) \cdots\left(T_{i-1}-\kappa_{i-N+m+1}\right) p_{i}\left(v_{i-1}\right) .
\end{aligned}
$$

Proof. The formula is true for $i=N-m$ by (12). Assume it holds for some $i$ then

$$
\begin{aligned}
& p_{i}\left(y_{i-1}\right)=\left(\frac{1-t}{1-z t^{N-i-1}}+\kappa_{i+m-N+1}\right) p_{i+1}\left(y_{i-1}\right)+\left(T_{i}-\kappa_{i+m-N+1}\right) p_{i+1}\left(y_{i}\right) \\
& p_{i}\left(v_{i-1}\right)=\left(\frac{1-t}{1-z t^{N-i-1}}+\kappa_{i+m-N+2}\right) p_{i+1}\left(v_{i-1}\right)+\left(T_{i}-\kappa_{i+m-N+2}\right) p_{i+1}\left(v_{i}\right),
\end{aligned}
$$

and

$$
\begin{aligned}
& \frac{1-t}{1-z t^{N-i-1}}+\kappa_{i+m-N+1}=\frac{1-z t^{m}}{\left(1-z t^{N-i-1}\right)[i+m-N+1]_{t}}, \\
& \frac{1-t}{1-z t^{N-i-1}}+\kappa_{i+m-N+2}=\frac{1-z t^{m+1}}{\left(1-z t^{N-i-1}\right)[i+m-N+2]_{t}} .
\end{aligned}
$$

Then $p_{i+1}$ satisfies the hypotheses of Lemma 8 for $N-m \leq j<i$ and

$$
\begin{aligned}
& \left(T_{N-m}-t\right) \cdots\left(T_{i-1}-\kappa_{i-N+m}\right) p_{i+1}\left(y_{i-1}\right) \\
& =(-1)^{i-N+m} \frac{[2]_{t}}{[1]_{t}} \frac{[3]_{t}}{[2]_{t}} \cdots \frac{[i-N+m+1]_{t}}{[i-N+m]_{t}} p_{i+1}\left(y_{N-m-1}\right) \\
& =(-1)^{i-N+m}[i-N+m+1]_{t} p_{i+1}\left(x^{(0)}\right) \\
& \left(T_{N-m-1}-t\right) \cdots\left(T_{i-1}-\kappa_{i-N+m+1}\right) p_{i}\left(v_{i-1}\right) \\
& =(-1)^{i-N+m+1} \frac{[2]_{t}}{[1]_{t}} \frac{[3]_{t}}{[2]_{t}} \cdots \frac{[i-N+m+2]_{t}}{[i-N+m+1]_{t}} p_{i+1}\left(v_{N-m-2}\right) \\
& =(-1)^{i-N+m+1}[i-N+m+2]_{t} p_{i+1}\left(x^{(0)}\right) .
\end{aligned}
$$

The first part of the right side of (14) combined with (16) gives

$$
\begin{aligned}
& \frac{1-z t^{m+1}}{1-z t^{m}} \frac{1-z t^{m}}{\left(1-z t^{N-i-1}\right)[i+m-N+1]_{t}}(-1)^{i-N+m}[i-N+m+1]_{t} p_{i+1}\left(x^{(0)}\right) \\
& =(-1)^{i-N+m} \frac{1-z t^{m+1}}{1-z t^{N-i-1}} p_{i+1}\left(x^{(0)}\right)
\end{aligned}
$$

which cancels out the first part of the right side of (15) combined with (18); note the factor $(-1)^{i-N+m+1}$. The terms that remain are exactly the claimed formula for $i+1$.

Proposition 11. $M_{\delta, E}\left(x^{(0)} ; \theta\right)=\frac{1-z t^{m+1}}{1-z t^{m}}\left(T_{N-m}-t\right) \cdots\left(T_{N-1}-\kappa_{m}\right) M_{\beta, E}\left(y_{N-1} ; \theta\right)$.

Proof. Set $i=N$ in (13). To complete the proof we need to show

$$
\left(T_{N-m-1}-t\right)\left(T_{N-m}-\kappa_{2}\right) \cdots\left(T_{N-1}-\kappa_{m+1}\right) p_{N}\left(v_{N-1}\right)=0 .
$$

By construction $\left(v_{N-1}\right)_{i}=t^{i}$ and $r_{\beta}(i)=i+1, \zeta_{\beta, E}(i)=t^{i+1-N}$ for $N-m-1 \leq i \leq$ $N-1$. This implies $\left(\boldsymbol{T}_{i}+1\right) p_{N}=0$ and $\left(T_{i}+1\right) p_{N}\left(v_{N-1}\right)=0$ for $N-m-1 \leq i<N-1$. Let $\psi_{1}=D\left(\theta_{N-m-1} \theta_{N-m} \cdots \theta_{N-1}\right)$ then $\left(T_{i}+1\right) \psi_{1}=0$ for $N-m-1 \leq i<N-1$. This property defines $\psi_{1}$ up to a multiplicative constant, and thus $p_{N}\left(v_{N-1}\right)=c \psi_{1}$ (because 
$\psi_{2}:=T_{N-m-1} T_{N-m} \cdots T_{N-1} \psi_{1}$ satisfies $\left(T_{j}+1\right) \psi_{2}=0$ for $N-m \leq j<N$ and thus $\left.\psi_{2}=c^{\prime} \tau_{E}\right)$. To set up an inductive argument let $\pi_{i}:=\theta_{N-m-1} \cdots \theta_{i-1} \theta_{i+1} \cdots \theta_{N}$ and set $f_{N}:=\pi_{N-1}, f_{N-j}:=\left(T_{N-j}-\kappa_{m+2-j}\right) f_{N-j+1}$ for $1 \leq j \leq m+1$. Then $T_{j} \pi_{i}=-\pi_{i}$ if $N-m-1 \leq j \leq i-2$ or $i+1 \leq j<N, T_{i-1} \pi_{i}=\pi_{i-1}$ and $T_{i} \pi_{i}=(t-1) \pi_{i}+t \pi_{i+1}$. Claim that

$$
f_{N-j}=\pi_{N-j}+\frac{1}{[m+2-j]_{t}} \sum_{i=0}^{j-1}(-1)^{i-j} t^{m+1-i} \pi_{N-i} .
$$

The first step is $f_{N-1}=\left(T_{N-1}-\kappa_{m+1}\right) \pi_{N}=\pi_{N-1}-\frac{t^{m+1}}{[m+1]_{t}} \pi_{N}$. Note $\kappa_{n}+1=$ $\frac{[n+1]_{t}}{[n]_{t}}$ for $n \geq 1$. Suppose the formula is true for some $j<m+1$, then

$$
\begin{aligned}
f_{N-j-1} & =\left(T_{N-j-1}-\kappa_{m+1-j}\right) f_{N-j}=\pi_{N-j-1}-\frac{t^{m+1-j}}{[m+1-j]_{t}} \pi_{N-j} \\
& +\frac{1}{[m+2-j]_{t}} \sum_{i}^{j-1}(-1)^{i-j} t^{m+1-i}\left(-\kappa_{m+1-j}-1\right) \pi_{N-i} \\
& =\pi_{N-j-1}+\frac{1}{[m+1-j]_{t}} \sum_{i}^{j}(-1)^{i-j-1} t^{m+1-i} \pi_{N-i} .
\end{aligned}
$$

This proves the formula. Set $j=m+1$ then $f_{N-m-1}=\sum_{i=0}^{m+1}(-1)^{m+1-i} t^{m+1-i} \pi_{N-i}$. By definition

$$
D\left(\pi_{N} \theta_{N}\right)=\sum_{j=N-m-1}^{N}(-1)^{j-N+m+1} t^{j-1} \pi_{j}=\sum_{i=0}^{m+1}(-1)^{m+1-i} t^{N-1-i} \pi_{N-i}
$$

and so $f_{N-m-1}=t^{m-N+2} D\left(\pi_{N} \theta_{N}\right)$. Now $p_{N}\left(v_{N-1}\right)=c \psi_{1}=c D\left(\pi_{N}\right)$. Thus

$$
\begin{aligned}
& \left(T_{N-m-1}-t\right)\left(T_{N-m}-\kappa_{2}\right) \cdots\left(T_{N-1}-\kappa_{m+1}\right) D\left(\pi_{N}\right) \\
& =D\left\{\left(T_{N-m-1}-t\right)\left(T_{N-m}-\kappa_{2}\right) \cdots\left(T_{N-1}-\kappa_{m+1}\right) \pi_{N}\right\}=D\left(f_{N-m-1}\right)=0,
\end{aligned}
$$

because $D^{2}=0$.

Next we consider the transition from $\alpha$ to $\beta$ (see (9)) with the affine step $M_{\beta, E}(x ; \theta)=$ $x_{N} \boldsymbol{w} M_{\alpha, E}(x ; \theta)$ (recall $\left.\boldsymbol{w} p(x ; \theta)=T_{N-1} \cdots T_{1} p\left(q x_{N}, x_{1}, \ldots, x_{N-1} ; \theta\right)\right)$. To get around the problem of evaluation at the $q$-shifted point we use $\xi_{1}=t^{1-N} \boldsymbol{T}_{1} \boldsymbol{T}_{2} \cdots \boldsymbol{T}_{N-1} w$ thus

$$
M_{\beta, E}(x ; \theta)=x_{N} t^{N-1}\left(\boldsymbol{T}_{N-1}^{-1} \cdots \boldsymbol{T}_{2}^{-1} \boldsymbol{T}_{1}^{-1} \xi_{1} M_{\alpha, E}\right)(x ; \theta)
$$

where $\xi_{1} M_{\alpha, E}=\zeta_{\alpha, E}(1) M_{\alpha, E}=q^{\lambda_{k}-1} t^{N-m-k} M_{\alpha, E}$. From the previous formula we see that we need to evaluate the right hand side at $x=y_{N-1}$ and apply $\left(T_{N-m}-t\right) \cdots\left(T_{N-1}-\kappa_{m}\right)$. Since $\zeta_{\alpha, E}(i)=t^{i-N}$ for $N-m \leq i \leq N$ it follows that $\left(\boldsymbol{T}_{i}+1\right) M_{\alpha, E}=0$ for $N-m \leq i<N$.

Definition 9. Let $r_{0}=M_{\alpha, E}$ and $r_{i}=t \boldsymbol{T}_{i}^{-1} r_{i-1}$ for $1 \leq i<N$.

The corresponding evaluation formula is

$$
r_{i}(x)=(1-t+b(x ; i)) r_{i-1}(x)+\left(T_{i}-b(x ; i)\right) r_{i-1}\left(x s_{i}\right) .
$$

Proposition 12. Suppose $1 \leq i \leq N-m-2$ then $r_{i}\left(x^{(0)}\right)=r_{0}\left(x^{(0)}\right)=M_{\alpha, E}\left(x^{(0)}\right)$.

Proof. From $\left(\boldsymbol{T}_{i}+1\right) M_{\alpha, E}=0$ for $N-m \leq i<N$ it follows that $\left(\boldsymbol{T}_{i}+1\right) r_{j}=0$ if $j<N-m-1$. By (20) $r_{\ell}\left(x^{(0)}\right)=r_{\ell-1}\left(x^{(0)}\right)+\left(T_{\ell}-t\right) r_{\ell-1}\left(x^{(0)} s_{\ell}\right)$. Suppose $\ell<$ 
$N-m-1$ then $x^{(0)} s_{\ell}$ satisfies $\left(x^{(0)} s_{\ell}\right)_{i}=t^{i-1}$ for $i \geq N-m$ so that $b\left(x^{(0)} s_{\ell} ; i\right)=t$ and $\left(T_{i}+1\right) r_{\ell-1}\left(x^{(0)} s_{\ell}\right)=0, r_{\ell-1}\left(x^{(0)} s_{\ell}\right)$ is a multiple of $\tau_{E}$ and $\left(T_{\ell}-t\right) r_{\ell-1}\left(x^{(0)} s_{\ell}\right)=0$. Thus, $r_{\ell}\left(x^{(0)}\right)=r_{\ell-1}\left(x^{(0)}\right)$ and this holds for $1 \leq \ell \leq N-m-2$.

Recall the points $y_{i}$ given by $y_{N-m-1}=x^{(0)}, y_{i}=y_{i-1} s_{i}$ for $N-m \leq i<N$. Define $\widetilde{y}_{i}=y_{i} s_{N-1} s_{N-2} \cdots s_{i+1}$. for $N-m-1 \leq i<N$. By the braid relations

$$
\begin{aligned}
\widetilde{y}_{i+1} s_{i+1} s_{i+2} & =\left(y_{i+1} s_{N-1} \cdots s_{i+2}\right) s_{i+1} s_{i+2}=y_{i+1} s_{N-1} \cdots s_{i+3} s_{i+1} s_{i+2} s_{i+1} \\
& =y_{i+1} s_{i+1} s_{N-1} \cdots s_{i+2} s_{i+1}=y_{i} s_{N-1} \cdots s_{i+1}=\widetilde{y}_{i} .
\end{aligned}
$$

These products are used in the proofs:

$$
\begin{aligned}
& P_{N-j}=\left(T_{N-m}-t\right)\left(T_{N-m+1}-\kappa_{2}\right) \cdots\left(T_{N-j}-\kappa_{m+1-j}\right) \\
& \widetilde{P}_{N-j}=\left(T_{N-1}-t\right)\left(T_{N-2}-\kappa_{2}\right) \cdots\left(T_{N-j}-\kappa_{j}\right) .
\end{aligned}
$$

If $i+1<j$ then $P_{N-j}$ commutes with $\widetilde{P}_{N-i}$.

Lemma 9. Suppose $\boldsymbol{T} f=-f$ for $N-j \leq i<N$ and $u_{i}=t^{i-1}$ for $N-j+1 \leq i \leq N$ then

$$
\widetilde{P}_{N-j} f\left(u s_{N-1} s_{N-2} \cdots s_{N-j}\right)=(-1)^{j}[j+1]_{t} f(u) .
$$

Proof. Let $\widetilde{u}^{(n)}=u s_{N-1} \cdots s_{N-n}$ then $\left(\widetilde{u}^{(n-1)}\right)_{N-n+1}=t^{N-1},\left(\widetilde{u}^{(n-1)}\right)_{N-n}=t^{N-n}$ and $b\left(\widetilde{u}^{(n-1)} ; N-n\right)=\kappa_{n-1}$. Thus

$$
\begin{aligned}
\left(T_{N-n}-\kappa_{n-1}\right) f\left(\widetilde{u}^{(n)}\right) & =\left(T_{N-n}-\kappa_{n-1}\right) f\left(\widetilde{u}^{(n-1)} s_{N-n}\right) \\
& =-\left(1+\kappa_{n-1}\right) f\left(\widetilde{u}^{(n-1)}\right)=-\frac{[n]_{t}}{[n-1]_{t}} f\left(\widetilde{u}^{(n-1)}\right) .
\end{aligned}
$$

Repeated application of this relation shows $\widetilde{P}_{N-j} f\left(u s_{N-1} s_{N-2} \cdots s_{N-j}\right)$ $=(-1)^{j} \frac{1}{[2]_{t}} \frac{[2]_{t}}{[3]_{t}} \cdots \frac{[j+1]_{t}}{[j]_{t}} f(u)$.

$$
\begin{aligned}
& \text { If } x_{i+1}=t^{j} x_{i i} \text { then } 1+b(x ; i)=\frac{[j+1]_{t}}{[j]_{t}}, 1-t+b(x ; i)=\frac{1}{[j]_{t}} \text { and } \\
& (1+b(x ; i))(t-b(x ; i))=\frac{t[j+1]_{t}[j-1]_{t}}{[j]_{t}^{2}} \text {. }
\end{aligned}
$$

Proposition 13. For $2 \leq j \leq m+1$

$$
\begin{aligned}
P_{N-1} r_{N-1}\left(y_{N-1}\right) & =t^{j-1} \frac{[m+1]_{t}[m+1-j]_{t}}{[m]_{t}[m+2-j]_{t}} P_{N-j} r_{N-j}\left(y_{N-j}\right) \\
& +(-1)^{j-1} \frac{t^{m}}{[m+2-j]_{t}} \widetilde{P}_{N-j+2} P_{N-j}\left(T_{N-j+1}-\kappa_{m}\right) r_{N-j}\left(\widetilde{y}_{N-j}\right) .
\end{aligned}
$$

Proof. Proceed by induction. By (11)

$$
\begin{gathered}
P_{N-1} r_{N-1}\left(y_{N-1}\right)=P_{N-2}\left(T_{N-1}-\kappa_{m}\right) r_{N-1}\left(y_{N-2} s_{N-1}\right) \\
=\frac{t[m+1]_{t}[m-1]_{t}}{[m]_{t}^{2}} P_{N-2} r_{N-2}\left(y_{N-2}\right)-\frac{t^{m}}{[m]_{t}} P_{N-2}\left(T_{N-1}-\kappa_{m}\right) r_{N-2}\left(y_{N-2} s_{N-1}\right)
\end{gathered}
$$


and $y_{N-1}=y_{N-2} s_{N-1}=\widetilde{y}_{N-1}$. Thus, the formula is valid for $j=2\left(\right.$ with $\left.\widetilde{P}_{N}=1\right)$. Suppose it holds for some $j \leq m$, then $b\left(y_{N-1-j} ; N-j\right)=\kappa_{m+1-j}$ and

$$
\begin{gathered}
P_{N-j} r_{N-j}\left(y_{N-j}\right)=P_{N-j-1}\left(T_{N-j}-\kappa_{m+1-j}\right) r_{N-j}\left(y_{N-1-j} s_{N-j}\right) \\
=\frac{t[m+2-j]_{t}[m-j]_{t}}{[m+1-j]_{t}^{2}} P_{N-j-1} r_{N-j-1}\left(y_{N-1-j}\right) \\
-\frac{t^{m+1-j}}{[m+1-j]_{t}} P_{N-j-1}\left(T_{N-j}-\kappa_{m+1-j}\right) r_{N-j-1}\left(y_{N-j}\right)
\end{gathered}
$$

Combine with formula (22) to obtain

$$
t^{j} \frac{[m+1]_{t}[m-j]_{t}}{[m]_{t}[m+1-j]_{t}} P_{N-j-1} r_{N-j-1}\left(y_{N-j-1}\right)-\frac{t^{m}[m+1]_{t}}{[m]_{t}[m+2-j]_{t}} P_{N-j} r_{N-j-1}\left(y_{N-j}\right) .
$$

For the part in (23) $b\left(\widetilde{y}_{N-j} ; N-j\right)=\kappa_{j-1}$ thus

$$
r_{N-j}\left(\widetilde{y}_{N-j}\right)=\frac{1}{[j-1]_{t}} r_{N-j-1}\left(\widetilde{y}_{N-j}\right)+\left(T_{N-j}-\kappa_{j-1}\right) r_{N-j-1}\left(\widetilde{y}_{N-j} s_{N-j}\right) .
$$

The first part leads to

$$
\begin{aligned}
& (-1)^{j-1} \frac{t^{m}}{[m+2-j]_{t}[j-1]_{t}} \widetilde{P}_{N-j+2} P_{N-j}\left(T_{N-j+1}-\kappa_{m}\right) r_{N-j-1}\left(\widetilde{y}_{N-j}\right) \\
& =(-1)^{j} \frac{[m+1]_{t}}{[m]_{t}} \frac{t^{m}}{[m+2-j]_{t}[j-1]_{t}} \widetilde{P}_{N-j+2} P_{N-j} r_{N-j-1}\left(\widetilde{y}_{N-j} S_{N-j+1}\right)
\end{aligned}
$$

because $b\left(\widetilde{y}_{N-j} s_{N-j+1} ; N-j+1\right)=\kappa_{m}$ and $\left(\boldsymbol{T}_{N-j+1}+1\right) r_{N-j-1}=0$. Then

$$
\begin{aligned}
\widetilde{P}_{N-j+2} P_{N-j} r_{N-j-1}\left(\widetilde{y}_{N-j} s_{N-j+1}\right) & =P_{N-j} \widetilde{P}_{N-j+2} r_{N-j-1}\left(y_{N-j} s_{N-1} \cdots s_{N-j+2}\right) \\
& =(-1)^{j}[j-1]_{t} P_{N-j} r_{N-j-1}\left(y_{N-j}\right)
\end{aligned}
$$

by Lemma 9, so combine to obtain $\frac{t^{m}[m+1]_{t}}{[m+2-j]_{t}[m]_{t}} P_{N-j} r_{N-j-1}\left(y_{N-j}\right)$ which cancels the second term in (24). The second part gives

$$
\begin{aligned}
& (-1)^{j-1} \frac{t^{m}}{[m+2-j]_{t}} \widetilde{P}_{N-j+2} P_{N-j}\left(T_{N-j+1}-\kappa_{m}\right)\left(T_{N-j}-\kappa_{j-1}\right) r_{N-j-1}\left(\widetilde{y}_{N-j} s_{N-j}\right) \\
& =\frac{(-1)^{j-1} t^{m}}{[m+2-j]_{t}} \widetilde{P}_{N-j+2} P_{N-j-1}\left(T_{N-j}-\kappa_{m-j+1}\right) \\
& \times\left(T_{N-j+1}-\kappa_{m}\right)\left(T_{N-j}-\kappa_{j-1}\right) r_{N-j-1}\left(\widetilde{y}_{N-j} s_{N-j}\right) \\
& =\frac{(-1)^{j-1} t^{m}}{[m+2-j]_{t}} \widetilde{P}_{N-j+2} P_{N-j-1}\left(T_{N-j+1}-\kappa_{j-1}\right) \\
& \times\left(T_{N-j}-\kappa_{m}\right)\left(T_{N-j+1}-\kappa_{m-j+1}\right) r_{N-j-1}\left(\widetilde{y}_{N-j} s_{N-j}\right) \\
& =\frac{(-1)^{j} t^{m}[m+2-j]_{t}}{[m+1-j]_{t}[m+2-j]_{t}} \widetilde{P}_{N-j+2} P_{N-j-1}\left(T_{N-j+1}-\kappa_{j-1}\right) \\
& \times\left(T_{N-j}-\kappa_{m}\right) r_{N-j-1}\left(\widetilde{y}_{N-j} s_{N-j} s_{-j+1}\right) \\
& =\frac{(-1)^{j} t^{m}}{[m+1-j]_{t}} \widetilde{P}_{N-j+1} P_{N-j-1}\left(T_{N-j}-\kappa_{m}\right) r_{N-j-1}\left(\widetilde{y}_{N-1-j}\right)
\end{aligned}
$$

by Lemma 7, formula (21) and $b\left(\widetilde{y}_{N-j} s_{N-j} ; N-j+1\right)=\kappa_{m-j+1}$. 
Proposition 14. $P_{N-1} r_{N-1}\left(y_{N-1}\right)=t^{m} M_{\alpha, E}\left(x^{(0)}\right)$.

Proof. Set $j=m+1$ in (22) thus

$$
\begin{aligned}
& P_{N-1} r_{N-1}\left(y_{N-1}\right)=(-1)^{m} t^{m} \widetilde{P}_{N-m+1}\left(T_{N-m}-\kappa_{m}\right) r_{N-m-1}\left(\widetilde{y}_{N-m-1}\right) \\
& =(-1)^{m} t^{m} \widetilde{P}_{N-m}\left\{\begin{array}{c}
1 \\
{ }^{[m+1]_{t}} r_{N-m-2}\left(\widetilde{y}_{N-m-1}\right) \\
+\left(T_{N-m-1}-\kappa_{m+1}\right) r_{N-m-2}\left(\widetilde{y}_{N-m-1} s_{N-m-1}\right)
\end{array}\right\}
\end{aligned}
$$

By Lemma $9 \widetilde{P}_{N-m} p_{r-m-2}\left(y_{N-m-1} s_{N-1} \cdots s_{N-m}\right)=(-1)^{m}[m+1]_{t} r_{N-m-2}\left(y_{N-m-1}\right)$. Furthermore

$$
\widetilde{y}_{N-m-1} s_{N-m+1}=\left(\begin{array}{ccc}
N-m-1 & N-m & N-m+1 \\
\ldots, & t^{N-1}, t^{N-m-2}, t^{N-m-1}, \ldots, t^{N-2}
\end{array}\right),
$$

and thus $\left(\boldsymbol{T}_{i}+1\right) r_{N-m-2}=0$ and by Lemma $1\left(T_{i}+1\right) r_{N-m-2}\left(\widetilde{y}_{N-m-1} s_{N-m-1}\right)=0$ for $N-m \leq i<N$. This implies $r_{N-m-2}\left(\widetilde{y}_{N-m-1} s_{N-m-1}\right)=c \tau_{E}$. However, $\widetilde{P}_{N-m-1} \tau_{E}=$ 0 and this is proved by an argument like the one used in Proposition 11. Let $\pi_{i}:=$ $\theta_{N-m-1} \cdots \theta_{i-1} \theta_{i+1} \cdots \theta_{N}$ and set $f_{0}:=\pi_{N-m-1}, f_{j}:=\left(T_{N-m-2+j}-\kappa_{m+2-j}\right) f_{j-1}$ for $1 \leq$ $j \leq m+1$. Claim

$$
f_{j}=t^{j} \pi_{N-m-1+j}+\frac{1}{[m+2-j]_{t}} \sum_{i=0}^{j-1}(-1)^{j-i} t^{i} \pi_{N-m-1+i}
$$

The first step is

$$
\begin{aligned}
f_{1} & =\left(T_{N-m-1}-\kappa_{m+1}\right) \pi_{N-m-1}=t \pi_{N-m}+\left(t-1-\kappa_{m+1}\right) \pi_{N-m--1} \\
& =t \pi_{N-m}-\frac{1}{[m+1]_{t}} \pi_{N-m-1} .
\end{aligned}
$$

Suppose the formula is true for some $j<m+1$ then

$$
\begin{aligned}
f_{j+1} & =\left(T_{N-m-1+j}-\kappa_{m+1-j}\right) f_{j}=t^{j+1} \pi_{N-m+j}+t^{j}\left(t-1-\kappa_{m+1-j}\right) \pi_{N-m-1+j} \\
& +\frac{1}{[m+2-j]_{t}}\left(-1-\kappa_{m+1-j}\right) \sum_{i=0}^{j-1}(-1)^{j-i} t^{i} \pi_{N-m-1+i} \\
& =t^{j+1} \pi_{N-m+j}-\frac{1}{[m+1-j]_{t}} t^{j} \pi_{N-m-1+j}+\frac{1}{[m+1-j]_{t}} \sum_{i=0}^{j-1}(-1)^{j+1-i} t^{i} \pi_{N-m-1+i},
\end{aligned}
$$

and this is the formula for $j+1$. Then $f_{m+1}=\sum_{i=0}^{m+1}(-1)^{m+1-i} t^{i} \pi_{N-m-1+i}$ and $D\left(\pi_{N} \theta_{N}\right)=$ $\sum_{i=N-m-1}^{N} t^{i-1}(-1)^{i-N+m+1} \pi_{i}$, and thus $f_{m+1}=(-1)^{m+1} t^{N-m-2} D\left(\pi_{N} \theta_{N}\right)=0$ and $D\left(f_{m+1}\right)=0$. As in Proposition 11 this implies $\widetilde{P}_{N-m-1} r_{N-m-2}\left(\widetilde{y}_{N-m-1} s_{N-m-1}\right)=0$, and this completes the proof. 


\subsection{Evaluation Formula for Type (0)}

Recall the intermediate steps:

$$
\begin{aligned}
V^{(0)}(\alpha) & =V^{(0)}\left(\lambda^{\prime}\right) \prod_{i=1}^{k-1} \frac{1-q^{\lambda_{i}-\lambda_{k}+1} t^{k-i}}{1-q^{\lambda_{i}-\lambda_{k}+1} t^{k-i+1}} \\
P_{N-1} r_{N-1}\left(y_{N-1}\right) & =t^{m} M_{\alpha, E}\left(x^{(0)} ; \theta\right) \\
M_{\beta, E}\left(y_{N-1} ; \theta\right) & =\zeta_{\alpha, E}(1)\left(y_{N-1}\right)_{N} r_{N-1}\left(y_{N-1}\right) \\
M_{\delta, E}\left(x^{(0)} ; \theta\right) & =\frac{1-q^{\lambda_{k}} t^{N-k+1}}{1-q^{\lambda_{k}} t^{N-k}} P_{N-1} M_{\beta, E}\left(y_{N-1} ; \theta\right) \\
V^{(0)}(\lambda) & =\frac{1-q^{\lambda_{k}} t^{N-m-k}}{1-q^{\lambda_{k} t}} V^{(0)}(\delta) .
\end{aligned}
$$

Proposition 15. Suppose $\lambda \in \mathcal{N}_{0}^{+}$satisfies $\lambda_{k} \geq 1$ and $\lambda_{i}=0$ for $i>k$ with $k<N-m$ then

$$
\begin{aligned}
V^{(0)}(\lambda) & =q^{\lambda_{k}-1} t^{2 N-m-k-1} \frac{\left(1-q^{\lambda_{k}} t^{N-m-k}\right)\left(1-q^{\lambda_{k}} t^{N-k+1}\right)}{\left(1-q^{\lambda_{k} t}\right)\left(1-q^{\lambda_{k}} t^{N-k}\right)} \\
& \times \prod_{i=1}^{k-1} \frac{1-q^{\lambda_{i}-\lambda_{k}+1} t^{k-i}}{1-q^{\lambda_{i}-\lambda_{k}+1} t^{k-i+1}} V^{(0)}\left(\lambda^{\prime}\right),
\end{aligned}
$$

where $\lambda_{j}^{\prime}=\lambda_{j}$ for $j \neq k$ and $\lambda_{k}^{\prime}=\lambda_{k}-1$

Proof. The leading factors are $t^{m} \zeta_{\alpha, E}(1)\left(y_{N-1}\right)_{N}=t^{m} q^{\lambda_{k}-1} t^{N-m-k} t^{N-m-1}$.

Corollary 1. Suppose $\lambda$ is as in the Proposition and $\lambda^{\prime \prime}$ satisfies $\lambda_{j}^{\prime \prime}=\lambda_{j}$ for $j \neq k$ and $\lambda_{k}^{\prime \prime}=0$ then

$$
\begin{aligned}
V^{(0)}(\lambda) & =q^{\left(\begin{array}{c}
{ }^{2} \\
2
\end{array}\right)} t^{\lambda_{k}(2 N-m-k-1)} \frac{\left(q t^{N-m-k} ; q\right)_{\lambda_{k}}\left(q t^{N-k+1} ; q\right)_{\lambda_{k}}}{(q t ; q)_{\lambda_{k}}\left(q t^{N-k} ; q\right)_{\lambda_{k}}} \\
& \times \prod_{i=1}^{k-1} \frac{\left(q t^{k-i} ; q\right)_{\lambda_{i}}\left(q t^{k-i+1} ; q\right)_{\lambda_{i}-\lambda_{k}}}{\left(q t^{k-i} ; q\right)_{\lambda_{i}-\lambda_{k}}\left(q t^{k-i+1} ; q\right)_{\lambda_{i}}}\left(\lambda^{\prime \prime}\right) .
\end{aligned}
$$

Proof. This uses

$$
\prod_{l=1}^{\lambda_{k}}\left(1-q^{\lambda_{i}-l+1} t^{n}\right)=\frac{\left(q t^{n} ; q\right)_{\lambda_{i}}}{\left(q t^{n} ; q\right)_{\lambda_{i}-\lambda_{k}}}
$$

with $n=k-i, k-i+1$.

This formula can now be multiplied out over $k$, starting with $\lambda=\mathbf{0}$, where $M_{0, E}(x ; \theta)=\tau_{E}(\theta)$.

Theorem 2. Suppose $\lambda \in \mathcal{N}_{0}^{+}$then

$$
V^{(0)}(\lambda)=q^{\beta(\lambda)} t^{e_{0}(\lambda)} \prod_{k=1}^{N-m-1} \frac{\left(q t^{N-k+1} ; q\right)_{\lambda_{k}}}{\left(q t^{N-k} ; q\right)_{\lambda_{k}}} \prod_{1 \leq i<j<N-m} \frac{\left(q t^{j-i+1} ; q\right)_{\lambda_{i}-\lambda_{j}}}{\left(q t^{j-i} ; q\right)_{\lambda_{i}-\lambda_{j}}}
$$

where $\beta(\lambda):=\sum_{i=1}^{N-m-1}\left(\begin{array}{c}\lambda_{i} \\ 2\end{array}\right)$ and $e_{0}(\lambda):=\sum_{i=1}^{N-m-1} \lambda_{i}(2 N-m-i-1)$ 
Proof. For $1 \leq k<N-m$ define $\lambda^{(k)}$ by $\lambda_{i}^{(k)}=\lambda_{i}$ for $1 \leq i \leq k$ and $\lambda_{i}^{(k)}=0$ for $i>k$. Formula (25) gives the value of $\rho_{k}:=V^{(0)}\left(\lambda^{(k)}\right) / V^{(0)}\left(\lambda^{(k-1)}\right)$. For fixed $i \leq k$ the products $(* ; q)_{\lambda_{i}}$ contribute

$$
\begin{aligned}
& \frac{\left(q t^{N-m-i} ; q\right)_{\lambda_{i}}\left(q t^{N-i+1} ; q\right)_{\lambda_{i}}}{(q t ; q)_{\lambda_{i}}\left(q t^{N-i} ; q\right)_{\lambda_{i}}} \prod_{j=i+1}^{k} \frac{\left(q t^{j-i} ; q\right)_{\lambda_{i}}}{\left(q t^{j-i+1} ; q\right)_{\lambda_{i}}} \\
& =\frac{\left(q t^{N-m-i} ; q\right)_{\lambda_{i}}\left(q t^{N-i+1} ; q\right)_{\lambda_{i}}}{(q t ; q)_{\lambda_{i}}\left(q t^{N-i} ; q\right)_{\lambda_{i}}} \frac{(q t ; q)_{\lambda_{i}}}{\left(q t^{k-i+1} ; q\right)_{\lambda_{i}}}=\frac{\left(q t^{N-m-i} ; q\right)_{\lambda_{i}}\left(q t^{N-i+1} ; q\right)_{\lambda_{i}}}{\left(q t^{k-i+1} ; q\right)_{\lambda_{i}}\left(q t^{N-i} ; q\right)_{\lambda_{i}}}
\end{aligned}
$$

to $\rho_{1} \rho_{2} \cdots \rho_{k}$ (the product telescopes). Each pair $(i, j)$ with $1 \leq i<j \leq k$ contributes

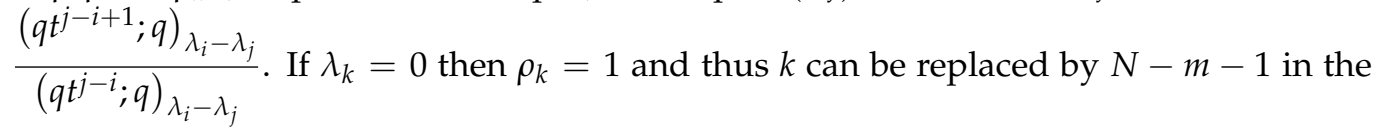
above formulas. The exponents on $q, t$ follow easily from $\rho_{k}$.

Remark 1. Recall the leading term of $M_{\lambda, E}(x ; \theta)$, namely $q^{\beta(\lambda)} t^{e(\lambda, E)} x^{\lambda} \tau_{E}(\theta)$, where $e(\lambda, E)=$ $\sum_{i=1}^{N} \lambda_{i}(N-i+c(i, E))$. By using $c(i, E)=N-m-i$ for $1 \leq i<N-m$ one finds that $e_{0}(\lambda)=\sum_{i=1}^{N-m-1} \lambda_{i}(N+c(i, E)-1)$ so that $e_{0}(\lambda)-e(\lambda, E)=\sum_{i=1}^{N-m-1} \lambda_{i}(i-1)=n(\lambda)$ and $\left(x^{(0)}\right)^{\lambda}=t^{n(\lambda)}$.

There is a generalized $(q, t, \lambda)$-Pochhammer symbol

$$
(a ; q, t)_{\lambda}:=\prod_{i=1}^{N}\left(a t^{1-i} ; q\right)_{\lambda_{i}}
$$

and the $k$-product in $(27)$ can be written as $\left(q t^{N} ; q, t\right)_{\lambda} /\left(q t^{N-1} ; q, t\right)_{\lambda}$. In a later section we will use a hook product formulation which incorporates a formula for $V^{(0)}(\alpha)$.

\subsection{From $\alpha$ to $\delta$ for Type (1)}

To adapt the results for type (0) to type (1) it almost suffices to interchange $m \leftrightarrow$ $N-m-1$ and replace $t$ by $t^{-1}$. However, there are signs and powers of $t$, and different formulas involving $\kappa_{-n}$ to worry about. The interchange occurs often enough to get a symbol:

Definition 10. Suppose $h(t, m)$ is a function of $t, m$ (possibly also depending on $\lambda$ or $\alpha$ ) then set $\Xi h(t, m)=h\left(t^{-1}, N-m-1\right)$

We will reuse some notations involving $y_{i}, p_{i}, r_{i}$ and so forth, with modified definitions (but conceptually the same). In this section, we will prove $M_{\delta, F}\left(x^{(1)}\right)=$ $q^{\lambda_{k}-1} t^{N-m-k} \frac{1-q^{\lambda_{k} t^{k-N-1}}}{1-q^{\lambda_{k} t^{k-N}}} M_{\alpha, F}\left(x^{(1)}\right)$. Start with $\delta$ (where $\delta_{i}=\lambda_{i}$ for $1 \leq i \leq k-$ $1, \delta_{N-m-1}=\lambda_{k}$ and $\delta_{i}=0$ otherwise). Let $\beta^{(m)}=\delta$ and $\beta^{(j)}=s_{j-1} \beta^{(j-1)}$ for $m+1 \leq j \leq N$ (so that $\beta^{(N)}=\beta$ in (9)). Abbreviate $z=\zeta_{\delta, F}(m)=\zeta_{\lambda, F}(k)=q^{\lambda_{k}} t^{k-1-m}$. If $m \leq i<N$ then $\zeta_{\beta^{(i+1), F}}(i+1)=z, \zeta_{\beta^{(i+1)}, F}(i)=t^{N-i-1}$. Set $p_{i}(x)=M_{\beta^{(i)}, F}(x ; \theta)$ for $m \leq i \leq N$, then

$$
\begin{aligned}
p_{i}(x) & =\left(\boldsymbol{T}_{i-1}+\frac{1-t}{1-z t^{i+1-N}}\right) p_{i+1}(x) \\
& =\left(b(x ; i)+\frac{1-t}{1-z t^{i+1-N}}\right) p_{i+1}(x)+\left(T_{i}-b(x ; i)\right) p_{i+1}\left(x s_{i}\right) .
\end{aligned}
$$


These are analogs of the type (0) definitions, with $m+1 \leq i \leq N-1$ :

$$
\begin{aligned}
y_{m} & =x^{(1)}, y_{m+1}=x^{(1)} s_{m+1}, y_{i}=y_{i-1} s_{i}, \\
v_{m} & =x^{(1)} s_{m}, v_{i}=v_{i-1} s_{i}, \\
P_{i} & :=\left(T_{m+1}+1\right)\left(T_{m+2}-\kappa_{-2}\right) \cdots\left(T_{i}-\kappa_{m-i}\right) .
\end{aligned}
$$

In more detail

$$
\begin{aligned}
& y_{i-1}=\left(\ldots, t^{1-m}, t^{-m-1}, \ldots, t^{-m}, t^{-i}, \ldots, t^{1-N}\right), b\left(y_{i-1} ; i\right)=\kappa_{m-i} \\
& v_{i-1}=\left(\ldots, t^{-m}, t^{-m-1}, \ldots, t^{1^{i}-m}, t^{-i}, \ldots, t^{1-N}\right), b\left(v_{i-1} ; i\right)=\kappa_{m-i-1} \cdot
\end{aligned}
$$

Recall $\zeta_{\delta^{(i)}, F}(i)=z, \zeta_{\delta^{(i)}, F}(i+1)=t^{N-i-1}$ for $m \leq i<N$. (The proof of the following is mostly the same as that for Proposition 10 except for signs and powers of $t$.)

Proposition 16. For $m+1 \leq i \leq N$

$$
\begin{aligned}
p_{m}\left(x^{(1)}\right) & =-t \frac{1-z t^{m-N}}{1-z t^{m+1-N}} P_{i-1} p_{i}\left(y_{i-1}\right) \\
& +\left(T_{m}+1\right)\left(T_{m+1}-\kappa_{-2}\right) \cdots\left(T_{i-1}-\kappa_{m-i}\right) p_{i}\left(v_{i-1}\right) .
\end{aligned}
$$

Proof. The transformation from $p_{i+1}$ to $p_{i}$ is in (28). Specialize to $i=m$ and $x=x^{(1)}$ so that $b(x ; i)=-1, x^{(1)} s_{m}=v_{m}$, and

$$
p_{m}\left(x^{(1)}\right)=-t \frac{1-z t^{m-N}}{1-z t^{m+1-N}} p_{m+1}\left(y_{m}\right)+\left(T_{m}+1\right) p_{m+1}\left(v_{m i}\right) .
$$

The values from (29) are $b\left(y_{i-1} ; i\right)=\kappa_{m-i}, b\left(v_{i-1} ; i\right)=\kappa_{m-i-1}, \zeta_{\delta^{(i)}, F}(i)=z$. Thus

$$
\begin{aligned}
& p_{i}\left(y_{i-1}\right)=\left(\frac{1-t}{1-z t^{i+1-N}}+\kappa_{m-i}\right) p_{i+1}\left(y_{i-1}\right)+\left(T_{i}-\kappa_{m-i}\right) p_{i+1}\left(y_{i-1} s_{i}\right) \\
& p_{i}\left(v_{i-1}\right)=\left(\frac{1-t}{1-z t^{i+1-N}}+\kappa_{m-i-1}\right) p_{i+1}\left(v_{i-1}\right)+\left(T_{i}-\kappa_{m-i-1}\right) p_{i+1}\left(v_{i-1} s_{i}\right),
\end{aligned}
$$

then

$$
\begin{aligned}
\frac{1-t}{1-z t^{i+1-N}}+\kappa_{m-i} & =-\frac{1-z t^{m-N+1}}{1-z t^{i+1-N}} \frac{t^{i-m}}{[i-m]_{t}}, \\
\frac{1-t}{1-z t^{i+1-N}}+\kappa_{m-i-1} & =-\frac{1-z t^{m-N}}{1-z t^{i+1-N}} \frac{t^{i+1-m}}{[i+1-m]_{t}}
\end{aligned}
$$

From the spectral vector of $p_{i+1}$ it follows that $\left(\boldsymbol{T}_{j}-t\right) p_{i+1}=0$ for $m \leq j<$ $i$ and $\left(T_{j}-b(x ; j)\right) p_{i+1}\left(x s_{j}\right)=(t-b(x ; j)) p_{i+1}(x)$. Thus, $\left(T_{i-1}-\kappa_{m+1-i}\right) p_{i+1}\left(y_{i-1}\right)=$ $\frac{[i-m]_{t}}{[i-m-1]_{t}} p_{i+1}\left(y_{i-2}\right)$ and

$$
\begin{aligned}
\left(T_{m+1}+1\right) \cdots\left(T_{i-1}-\kappa_{m+1-i}\right) p_{i+1}\left(y_{i-1}\right) & =[i-m]_{t} p_{i+1}\left(y_{m}\right) \\
\left(T_{m}+1\right)\left(T_{m+1}-\kappa_{-2}\right) \cdots\left(T_{i-1}-\kappa_{m-i}\right) p_{i+1}\left(v_{i-1}\right) & =[i+1-m]_{t} p_{i+1}\left(x^{(1)}\right) .
\end{aligned}
$$

Then $p_{i+1}\left(y_{m}\right)$ appears in the expression for $p_{m}\left(x^{(1)}\right)$ with factor

$$
\left(-t \frac{1-z t^{m-N}}{1-z t^{m+1-N}}\right)\left(-\frac{1-z t^{m-N+1}}{1-z t^{i+1-N}} \frac{t^{i-m}}{[i-m]_{t}}\right)[i-m]_{t}=t^{i+1-m} \frac{1-z t^{m-N}}{1-z t^{i+1-N}}
$$


and $p_{i+1}\left(x^{(1)}\right)$ with factor $-\frac{1-z t^{m-N}}{1-z t^{i+1-N}} \frac{t^{i+1-m}}{[i+1-m]_{t}}[i+1-m]_{t}$ and the two cancel out $\left(y_{m}=x^{(1)}\right)$. This proves the inductive step.

Proposition 17. $M_{\delta, F}\left(x^{(1)}\right)=-t \frac{1-z t^{m-N}}{1-z t^{m+1-N}} P_{N-1} p_{N}\left(y_{N-1}\right)$.

Proof. Set $i=N$ in (30). Claim $\left(T_{m}+1\right)\left(T_{m+1}-\kappa_{-2}\right) \cdots\left(T_{N-1}-\kappa_{m-N}\right) p_{N}\left(v_{N-1}\right)=0$. From $\left(v_{N-1}\right)_{i}=t^{-i}$ and $\zeta_{\beta, F}(i)=t^{N-i-1}$ for $m \leq i \leq N-1$ it follows that $\left(T_{i}-t\right) p_{N}\left(v_{N-1}\right)$ $=0$ for $m \leq i \leq N-2$. This implies $p_{N}\left(v_{N-1}\right)=c M\left(\theta_{1} \theta_{2} \cdots \theta_{m-1} \theta_{N}\right)$ for some constant (similarly to the argument in Proposition $11 h=T_{m} T_{m+1} \cdots T_{N-1} M\left(\theta_{1} \cdots \theta_{m-1} \theta_{N}\right)$ satisfies $\left(T_{i}-t\right) h=0$ for $m+1 \leq i \leq N-1$ implying $\left.h=c^{\prime} \tau_{F}\right)$. Let $g=\theta_{1} \theta_{2} \cdots \theta_{m-1}$ and $f_{0}=g \theta_{N}$ then define $f_{i}=\left(T_{N-i}-\kappa_{m-N+i-1}\right) f_{i-1}$ for $1 \leq i \leq N-m$. Use induction to show

$$
f_{i}=t^{i} g \theta_{N-i}+\frac{t^{N-m}}{[N-m+1-i]_{t}} \sum_{j=0}^{i-1} g \theta_{N-j} .
$$

The start is

$$
\begin{aligned}
f_{1} & =g \theta_{N}=\left(T_{N-1}+\frac{1}{[N-m]_{t}}\right) g \theta_{N}=\operatorname{tg} \theta_{N-1}+\left(t-1+\frac{1}{[N-m]_{t}}\right) g \theta_{N} \\
& =\operatorname{tg} \theta_{N-1}+\frac{t^{N-m}}{[N-m]_{t}} g \theta_{N} .
\end{aligned}
$$

Assume the formula is true for some $i<N-m$ then

$$
\begin{aligned}
f_{i+1} & =\left(T_{N-i-1}-\kappa_{m-N+i}\right) f_{i}=t^{i+1} g \theta_{N-i-1}+t^{i}\left(t-1+\frac{1}{[N-m-i]_{t}}\right) g \theta_{N-i} \\
& +\frac{t^{N-m}}{[N-m+1-i]_{t}}\left\{t+\frac{1}{[N-m-i]_{t}}\right\} \sum_{j=0}^{i-1} g \theta_{N-j} \\
& =t^{i+1} g \theta_{N-i-1}+\frac{t^{N-m}}{[N-m-i]_{t}} \sum_{j=0}^{i} g \theta_{N-j}
\end{aligned}
$$

(because $\left.t+\frac{1}{[n]_{t}}=\frac{[n+1]_{t}}{[n] t}\right)$. Thus, $f_{N-m}=t^{N-m} g \sum_{j=0}^{N-m} \theta_{N-j}=(-1)^{m-1} t^{N-m} M(g)$ and $M\left(f_{N-m}\right)=0$.

Next we consider the transition from $\alpha$ to $\beta$ (see (9)) with the affine step $M_{\beta, F}(x ; \theta)=$ $x_{N} w M_{\alpha, F}(x ; \theta)$ and as before the calculation is based on the formula

$$
M_{\beta, F}(x ; \theta)=x_{N} t^{N-1}\left(\boldsymbol{T}_{N-1}^{-1} \cdots \boldsymbol{T}_{2}^{-1} \boldsymbol{T}_{1}^{-1} \xi_{1} M_{\alpha, F}\right)(x ; \theta)
$$

where $\xi_{1} M_{\alpha, F}=\zeta_{\alpha, F}(1) M_{\alpha, F}=q^{\lambda_{k}-1} t^{k-m-1} M_{\alpha, F}$. From the previous formula we see that we need to evaluate $P_{N-1} M_{\beta, F}\left(y_{N-1}\right)$. Since $\zeta_{\alpha, F}(i)=t^{N-i}$ for $m+1 \leq i \leq N$ it follows that $\left(\boldsymbol{T}_{i}-t\right) M_{\alpha, F}=0$ for $m+1 \leq i<N$.

Definition 11. Let $r_{0}=M_{\alpha, F}$ and $r_{i}=t \boldsymbol{T}_{i}^{-1} r_{i-1}$ for $1 \leq i<N$.

Proposition 18. Suppose $1 \leq i \leq m-1$ then $r_{i}\left(x^{(1)}\right)=(-t)^{i} r_{0}\left(x^{(1)}\right)=(-t)^{i} M_{\alpha, F}\left(x^{(1)}\right)$.

Proof. From $\left(\boldsymbol{T}_{i}-t\right) M_{\alpha, F}=0$ for $m+1 \leq i<N$ it follows that $\left(\boldsymbol{T}_{i}-t\right) r_{j}=0$ if $j<m$. By (20) $r_{\ell}\left(x^{(1)}\right)=-t r_{\ell-1}\left(x^{(1)}\right)+\left(T_{\ell}+1\right) r_{\ell-1}\left(x^{(1)} s_{\ell}\right)$. Suppose $\ell<N-$ $m-1$ then $x^{(0)} s_{\ell}$ satisfies $\left(x^{(1)} s_{\ell}\right)_{i}=t^{-i-}$ for $i \geq m+1$ so that $b\left(x^{(0)} s_{\ell} ; i\right)=-1$ and 
$\left(T_{i}-t\right) r_{\ell-1}\left(x^{(1)} s_{\ell}\right)=0, r_{\ell-1}\left(x^{(1)} s_{\ell}\right)$ is a multiple of $\tau_{F}$ and $\left(T_{\ell}+1 t\right) r_{\ell-1}\left(x^{(1)} s_{\ell}\right)=0$. Thus, $r_{\ell}\left(x^{(0)}\right)=-\operatorname{tr}_{\ell-1}\left(x^{(0)}\right)$ and this holds for $1 \leq \ell \leq m-1$.

Similarly to the type (0) computations let

$$
\begin{aligned}
\widetilde{P}_{N-j} & :=\left(T_{N-1}+1\right)\left(T_{N-2}-\kappa_{-2}\right) \cdots\left(T_{N-j}-\kappa_{-j}\right) \\
\widetilde{y}_{N-j-1} & :=y_{N-j-1} s_{N-1} s_{N-2} \cdots s_{N-j} .
\end{aligned}
$$

Lemma 10. Suppose $\boldsymbol{T}_{i} f=t f$ for $N-j \leq i<N$ and $u_{i}=c t^{1-i}$ for $N-j+1 \leq i \leq N$ then

$$
\left(T_{N-1}+1\right)\left(T_{N-2}-\kappa_{-2}\right) \cdots\left(T_{N-j}-\kappa_{-j}\right) f\left(u s_{N-1} s_{N-2} \cdots s_{N-j}\right)=[j+1]_{t} f(u) .
$$

Proof. Let $\widetilde{u}^{(k)}=u s_{N-1} \cdots s_{N-k}$ then $\left(\widetilde{u}^{(k-1)}\right)_{N-k+1}=t^{1-N},\left(\widetilde{u}^{(k-1)}\right)_{N-k}=t^{k-N}$ and $b\left(\widetilde{u}^{(k-1)} ; N-k\right)=\kappa_{1-k}$. Thus

$$
\begin{aligned}
\left(T_{N-k}-\kappa_{1-k}\right) f\left(\widetilde{u}^{(k)}\right) & =\left(T_{N-k}-\kappa_{1-k}\right) f\left(\widetilde{u}^{(k-1)} s_{N-k}\right) \\
& =\left(t-\kappa_{1-k}\right) f\left(\widetilde{u}^{(k-1)}\right)=\frac{[k]_{t}}{[k-1]_{t}} .
\end{aligned}
$$

Repeated application of this formula shows

$$
\begin{aligned}
\left(T_{N-1}+1\right) \cdots\left(T_{N-j}-\kappa_{-j}\right) f\left(u s_{N-1} s_{N-2} \cdots s_{N-j}\right) & =\frac{1}{[2]_{t}} \frac{[2]_{t}}{[3]_{t}} \cdots \frac{[j+1]_{t}}{[j]_{t}} f(u) \\
& =[j+1]_{t} f(u) .
\end{aligned}
$$

Proposition 19. For $2 \leq j \leq N-m$

$$
\begin{aligned}
P_{N-1} r_{N-1}\left(y_{N-1}\right) & =t^{j-1} \frac{[N-m]_{t}[N-m-j]_{t}}{[N-m-1]_{t}[N-m+1-j]_{t}} P_{N-j} r_{N-j}\left(y_{N-j}\right) \\
& +\frac{1}{[N-m+1-j]_{t}} \widetilde{P}_{N-j+2} P_{N-j}\left(T_{N-j+1}-\kappa_{m+1-N}\right) r_{N-j}\left(\widetilde{y}_{N-j}\right) .
\end{aligned}
$$

Proof. Proceed by induction. By (11)

$$
\begin{aligned}
P_{N-1} r_{N-1}\left(y_{N-1}\right) & =P_{N-2}\left(T_{N-1}-\kappa_{m-N+1}\right) r_{N-1}\left(y_{N-2} s_{N-1}\right) \\
& =\frac{t[N-m]_{t}[N-m-2]_{t}}{[N-m-1]_{t}^{2}} P_{N-2} r_{N-2}\left(y_{N-2}\right) \\
& +\frac{1}{[N-m-1]_{t}} P_{N-2}\left(T_{N-1}-\kappa_{m+1-N}\right) r_{N-2}\left(y_{N-2} s_{N-1}\right)
\end{aligned}
$$

and $y_{N-1}=y_{N-2} s_{N-1}=\widetilde{y}_{N-2}$. Thus, the formula is valid for $j=2\left(\right.$ with $\left.\widetilde{P}_{N}=1\right)$. Suppose it holds for some $j \leq N-m-2$, then $b\left(y_{N-1-j} ; N-j\right)=\kappa_{j+m-N}$ and

$$
\begin{aligned}
P_{N-j} r_{N-j}\left(y_{N-j}\right) & =P_{N-j-1}\left(T_{N-j}-\kappa_{j+m-N}\right) r_{N-j}\left(y_{N-j-1} s_{N-j}\right) \\
& =\frac{t[N-m+1-j]_{t}[N-m-1-j]_{t}}{[N-m-j]_{t}^{2}} P_{N-j-1} r_{N-j-1}\left(y_{N-j-1}\right) \\
& +\frac{1}{[N-m-j]_{t}} P_{N-j-1}\left(T_{N-j}-\kappa_{j+m-N}\right) r_{N-j-1}\left(y_{N-j}\right) .
\end{aligned}
$$


Combine with formula (31) to obtain

$$
\begin{aligned}
& t^{j} \frac{[N-m]_{t}[N-m-1-j]_{t}}{[N-m-1]_{t}[N-m-j]_{t}} P_{N-j-1} r_{N-j-1}\left(y_{N-j-1}\right) \\
& +\frac{t^{j-1}[N-m]_{t}}{[N-m-1]_{t}[N-m+1-j]_{t}} P_{N-j} r_{N-j-1}\left(y_{N-j}\right) .
\end{aligned}
$$

For the second line (32) $b\left(\widetilde{y}_{N-j} ; N-j\right)=\kappa_{1-j}$ thus

$$
r_{N-j}\left(\widetilde{y}_{N-j}\right)=-\frac{t^{j-1}}{[j-1]_{t}} r_{N-j-1}\left(\widetilde{y}_{N-j}\right)+\left(T_{N-j}-\kappa_{1-j}\right) r_{N-j-1}\left(\widetilde{y}_{N-j} s_{N-j}\right) .
$$

The first part leads to

$$
\begin{aligned}
& -\frac{t^{j-1}}{[N-m+1-j]_{t}[j-1]_{t}} \widetilde{P}_{N-j+2} P_{N-j}\left(T_{N-j+1}-\kappa_{m+1-N}\right) r_{N-j-1}\left(\widetilde{y}_{N-j}\right) \\
& =-\frac{[N-m]_{t}}{[N-m-1]_{t}} \frac{t^{j-1}}{[N-m+1-j]_{t}[j-1]_{t}} \widetilde{P}_{N-j+2} P_{N-j} r_{N-j-1}\left(\widetilde{y}_{N-j} s_{N-j+1}\right)
\end{aligned}
$$

because $b\left(\widetilde{y}_{N-j} s_{N-j+1} ; N-j+1\right)=\kappa_{m+1-N}$ and $\left(\boldsymbol{T}_{N-j+1}-t\right) r_{N-j-1}=0$. Then

$$
\begin{aligned}
\widetilde{P}_{N-j+2} P_{N-j} r_{N-j-1}\left(\widetilde{y}_{N-j} s_{N-j+1}\right) & =P_{N-j} \widetilde{P}_{N-j+2} r_{N-j-1}\left(y_{N-j} s_{N-1} \cdots s_{N-j+2}\right) \\
& =[j-1]_{t} P_{N-j} r_{N-j-1}\left(y_{N-j}\right)
\end{aligned}
$$

by Lemma 10, so combine to obtain $\frac{[N-m]_{t}}{[N-m-1]_{t}} \frac{(-1) t^{j-1}}{[N-m+1-j]_{t}} P_{N-j} r_{N-j-1}\left(y_{N-j}\right)$ which cancels the second term in (33). The second part gives (using the braid relations in (7))

$$
\begin{aligned}
& \frac{1}{[N-m+1-j]_{t}} \widetilde{P}_{N-j+2} P_{N-j}\left(T_{N-j+1}-\kappa_{m+1-N}\right)\left(T_{N-j}-\kappa_{1-j}\right) r_{N-j-1}\left(\widetilde{y}_{N-j} s_{N-j}\right) \\
& =\frac{1}{[N-m+1-j]_{t}} \widetilde{P}_{N-j+2} P_{N-j-1} \\
& \times\left(T_{N-j}-\kappa_{m+j-N}\right)\left(T_{N-j+1}-\kappa_{m+1-N}\right)\left(T_{N-j}-\kappa_{1-j}\right) r_{N-j-1}\left(\widetilde{y}_{N-j} s_{N-j}\right) \\
& =\frac{1}{[N-m+1-j]_{t}} \widetilde{P}_{N-j+2} P_{N-j-1} \\
& \times\left(T_{N-j+1}-\kappa_{1-j}\right)\left(T_{N-j}-\kappa_{m+1-N}\right)\left(T_{N-j+1}-\kappa_{m+j-N}\right) r_{N-j-1}\left(\widetilde{y}_{N-j} s_{N-j}\right) \\
& =\frac{[N-m+1-j]_{t}}{[N-m+1-j]_{t}[N-m-j]_{t}} \widetilde{P}_{N-j+2} P_{N-j-1} \\
& \times\left(T_{N-j+1}-\kappa_{1-j}\right)\left(T_{N-j}-\kappa_{m+1-N}\right) r_{N-j-1}\left(\widetilde{y}_{N-j} s_{N-j} s_{N-j+1}\right) \\
& =\frac{1}{[N-m-j]_{t}} \widetilde{P}_{N-j+1} P_{N-j-1}\left(T_{N-j}-\kappa_{m+1-N}\right) r_{N-j-1}\left(\widetilde{y}_{N-j-1}\right)
\end{aligned}
$$

by $(21)$ and $b\left(\widetilde{y}_{N-j-1} ; N-j+1\right)=\kappa_{m+j-N}$.

Proposition 20. $P_{N-1} r_{N-1}\left(y_{N-1}\right)=(-1)^{m} t^{N-1} M_{\beta, F}\left(x^{(1)} ; \theta\right)$.

Proof. Set $j=N-m$ in (31)

$$
P_{N-1} r_{N-1}\left(y_{N-1}\right)=\widetilde{P}_{m+2}\left(T_{m+1}-\kappa_{m+1-N}\right) r_{m}\left(\widetilde{y}_{m}\right)=\widetilde{P}_{m+1} r_{m}\left(\widetilde{y}_{m}\right)
$$


and $b\left(\widetilde{y}_{m} ; m\right)=\kappa_{m-N}\left(\right.$ note $\left.\widetilde{y}_{m}=x^{(1)} s_{N-1} s_{N-2} \cdots s_{m+1}\right)$ thus

$$
\widetilde{P}_{m+1} r_{m}\left(\widetilde{y}_{m}\right)=-\frac{t^{N-m}}{[N-m]_{t}} \widetilde{P}_{m+1} r_{m-1}\left(\widetilde{y}_{m}\right)+\widetilde{P}_{m+1}\left(T_{m}-\kappa_{m-N}\right) r_{m-1}\left(\widetilde{y}_{m} s_{m}\right) .
$$

Now $\widetilde{y}_{m}=\left(\ldots, t^{1-m}, t^{1-N}, t^{-m}, \ldots, t^{2-N}\right)$ thus $\widetilde{y}_{m}$ satisfies the hypothesis of Lemma 10 with $j=N-m-1$ and

$$
\begin{aligned}
& \left(T_{N-1}+1\right)\left(T_{N-2}-\kappa_{-2}\right) \cdots\left(T_{m+1}-\kappa_{m+1-N}\right) r_{m-1}\left(x^{(1)} s_{N-1} s_{N-2} \cdots s_{m+1}\right) \\
& =[N-m]_{t} r_{m-1}\left(x^{(1)}\right) .
\end{aligned}
$$

Since $\widetilde{y}_{m} s_{m}=\left(\ldots, t^{1-N}, t^{(-m+1)}, t^{-m}, \ldots t^{2-N}\right)$ and $\left(\boldsymbol{T}_{i}-t\right) r_{m-1}=0$ for $m+1 \leq i<$ $N$ it follows that $\left(T_{i}-t\right) r_{m-1}\left(\widetilde{y}_{m} s_{m}\right)=0$ for the same $i$ values and hence $r_{m-1}\left(\widetilde{y}_{m} s_{m}\right)=c \tau_{F}$ (with $F=\{1,2, \ldots, m\}$ because $m+1, m+2, \ldots, N$ lie in the same row of $Y_{F}$ ). Take $\tau_{F}=M\left(\theta_{1} \cdots \theta_{m}\right)$ and $g=\theta_{1} \cdots \theta_{m-1}$ then

$$
\begin{aligned}
\left(T_{m}-\kappa_{m-N}\right)\left(g \theta_{m}\right) & =g \theta_{m+1}+\frac{1}{[N-m]_{t}} g \theta_{m} \\
\left(T_{m+1}-\kappa_{m+1-N}\right)\left(T_{m}-\kappa_{m-N}\right)\left(g \theta_{m}\right) & =g \theta_{m+2}+\frac{1}{[N-m-1]_{t}} g \theta_{m+1} \\
& +\left(t+\frac{1}{[N-m-1]_{t}}\right) \frac{1}{[N-m]_{t}} g \theta_{m} \\
& =g \theta_{m+2}+\frac{1}{[N-m-1]_{t}} g\left(\theta_{m+1}+\theta_{m}\right),
\end{aligned}
$$

because $t+\frac{1}{[j-1]_{t}}=\frac{[j]_{t}}{[j-1]_{t}}$. Continue this process to obtain

$$
\left(T_{N-1}-\kappa_{-1}\right) \cdots\left(T_{m}-\kappa_{m-N}\right)\left(g \theta_{m}\right)=g\left(\theta_{N}+\cdots+\theta_{m}\right)=(-1)^{m-1} M(g)
$$

thus $\left(T_{N-1}-\kappa_{-1}\right) \cdots\left(T_{m}-\kappa_{m-N}\right) r_{m-1}\left(\widetilde{y}_{m} s_{m}\right)=0$ because $M^{2}=0$. Thus, $P_{N-1} r_{N-1}\left(y_{N-1}\right)$ $=-t^{N-m} r_{m-1}\left(x^{(1)}\right)=(-1)^{m} t^{N-1} r_{0}\left(x^{(1)}\right)$.

\subsection{Evaluation Formula for Type (1)}

Recall the intermediate steps:

$$
\begin{aligned}
V^{(1)}(\alpha) & =(-t)^{1-k} \prod_{i=1}^{k-1} \frac{1-q^{\lambda_{i}-\lambda_{k}+1} t^{i-i}}{1-q^{\lambda_{i}-\lambda_{k}+1} t^{i-k-1}} V^{(1)}\left(\lambda^{\prime}\right) \\
P_{N-1} r_{N-1}\left(y_{N-1}\right) & =(-1)^{m} t^{N-1} M_{\alpha, F}\left(x^{(1)} ; \theta\right) \\
M_{\beta, F}\left(y_{N-1} ; \theta\right) & =\zeta_{\alpha, F}(1)\left(y_{N-1}\right)_{N} r_{N-1}\left(y_{N-1}\right) \\
M_{\delta, F}\left(x^{(1)} ; \theta\right) & =-t \frac{1-q^{\lambda_{k}} t^{k-N-1}}{1-q^{\lambda_{k}} t^{k-N}} P_{N-1} M_{\beta, F}\left(y_{N-1} ; \theta\right) \\
V^{(1)}(\lambda) & =(-t)^{m-k} \frac{1-q^{\lambda_{k}} t^{k-m-1}}{1-q^{\lambda_{k} t^{-1}}} V^{(1)}(\delta) .
\end{aligned}
$$


Proposition 21. Suppose $\lambda \in \mathcal{N}_{1}^{+}$satisfies $\lambda_{k} \geq 1$ and $\lambda_{i}=0$ for $i>k$ with $k \leq m$ then

$$
\begin{aligned}
V^{(1)}(\lambda) & =q^{\lambda_{k}-1} t^{N-m-k} \frac{\left(1-q^{\lambda_{k}} t^{k-m-1}\right)\left(1-q^{\lambda_{k}} t^{k-N-1}\right)}{\left(1-q^{\lambda_{k} t^{-1}}\right)\left(1-q^{\lambda_{k} t^{k-N}}\right)} \\
& \times \prod_{i=1}^{k-1} \frac{1-q^{\lambda_{i}-\lambda_{k}+1} t^{i-k}}{1-q^{\lambda_{i}-\lambda_{k}+1} t^{i-k-1}} V^{(1)}\left(\lambda^{\prime}\right),
\end{aligned}
$$

where $\lambda_{j}^{\prime}=\lambda_{j}$ for $j \neq k$ and $\lambda_{k}^{\prime}=\lambda_{k}-1$.

Proof. The leading factors are $t^{N+m-2 k+1} \zeta_{\alpha, F}(1)\left(y_{N-1}\right)_{N}=t^{N-m-k} q^{\lambda_{k}-1}$, since $\zeta_{\alpha, F}(1)=$ $q^{\lambda_{k}-1} t^{k-1-m}$ and $\left(y_{N-1}\right)_{N}=t^{-m}$.

Corollary 2. Suppose $\lambda$ is as in the Proposition and $\lambda^{\prime \prime}$ satisfies $\lambda_{j}^{\prime \prime}=\lambda_{j}$ for $j \neq k$ and $\lambda_{k}^{\prime \prime}=0$ then

$$
\begin{aligned}
V^{(1)}(\lambda) & =q^{\left(\lambda_{k}\right)} t^{\lambda_{k}(N-m-k)} \frac{\left(q t^{k-m-1} ; q\right)_{\lambda_{k}}\left(q t^{k-N-1} ; q\right)_{\lambda_{k}}}{\left(q t^{-1} ; q\right)_{\lambda_{k}}\left(q t^{k-N} ; q\right)_{\lambda_{k}}} \\
& \times \prod_{i=1}^{k-1} \frac{\left(q t^{i-k} ; q\right)_{\lambda_{i}}\left(q t^{i-k-1} ; q\right)_{\lambda_{i}-\lambda_{k}}}{\left(q t^{i-k} ; q\right)_{\lambda_{i}-\lambda_{k}}\left(q t^{i-k-1} ; q\right)_{\lambda_{i}}} V^{(1)}\left(\lambda^{\prime \prime}\right) .
\end{aligned}
$$

Proof. This uses formula (26).

This formula can now be multiplied out over $k$, starting with $\lambda=\mathbf{0}$, where $M_{0, F}(x ; \theta)=\tau_{F}(\theta)$.

Theorem 3. Suppose $\lambda \in \mathcal{N}_{1}^{+}$then

$$
V^{(1)}(\lambda)=q^{\beta(\lambda)} t^{e_{1}(\lambda)} \prod_{k=1}^{m} \frac{\left(q t^{k-N-1} ; q\right)_{\lambda_{k}}}{\left(q t^{k-N} ; q\right)_{\lambda_{k}}} \prod_{1 \leq i<j \leq m} \frac{\left(q t^{i-1-1} ; q\right)_{\lambda_{i}-\lambda_{j}}}{\left(q t^{i-j} ; q\right)_{\lambda_{i}-\lambda_{j}}}
$$

where $\beta(\lambda):=\sum_{i=1}^{N-m-1}\left(\begin{array}{c}\lambda_{i} \\ 2\end{array}\right)$ and $e_{1}(\lambda):=\sum_{i=1}^{m} \lambda_{i}(N-m-i)$.

Proof. This is the same argument used in Theorem 2 by the application of $\Xi$.

Remark 2. Recall the leading term of $M_{\lambda, F}(x ; \theta)$, namely $q^{\beta(\lambda)} t^{e(\lambda, F)} x^{\lambda} \tau_{F}(\theta)$, where $e(\lambda, F)=$ $\sum_{i=1}^{N} \lambda_{i}(N-i+c(i, F))$. By using $c(i, F)=i-m-1$ for $1 \leq i \leq m$ one finds that $e_{1}(\lambda)=$ $\sum_{i=1}^{m} \lambda_{i}(N+c(i, F)-2 i+1)$ so that $e_{1}(\lambda)-e(\lambda, F)=-\sum_{i=1}^{m} \lambda_{i}(i-1)=-n(\lambda)$ and $\left(x^{(1)}\right)^{\lambda}=t^{-n(\lambda)}$.

\section{Hook Product Formulation}

Recall the definition of the $(q, t)$-hook product

$$
h_{q, t}(a ; \lambda)=\prod_{(i, j) \in \lambda}\left(1-a q^{\operatorname{arm}(i, j ; \lambda)} t^{\operatorname{leg}(i, j ; \lambda)}\right),
$$

where $\operatorname{arm}(i, j ; \lambda)=\lambda_{i}-j$ and $\operatorname{leg}(i, j ; \lambda)=\#\left\{l: i<l \leq \ell(\lambda), j \leq \lambda_{l}\right\}$, where the length of $\lambda$ is $\ell(\lambda)=\max \left\{i: \lambda_{i} \geq 1\right\}$. The terminology refers to the Ferrers diagram of $\lambda$ which consists of boxes at $\left\{(i, j): 1 \leq i \leq \ell(\lambda), 1 \leq j \leq \lambda_{i}\right\}$. 
Proposition 22. Suppose $\lambda \in \mathbb{N}_{0}^{N,+}$ and $\ell(\lambda) \leq L$ for some fixed $L \leq N$ then

$$
\prod_{1 \leq i<j \leq L} \frac{\left(q t^{j-i} ; q\right)_{\lambda_{i}-\lambda_{j}}}{\left(q t^{j-i+1} ; q\right)_{\lambda_{i}-\lambda_{j}}}=h_{q, t}(q t ; \lambda) \prod_{i=1}^{L}\left(q t^{L-i+1} ; q\right)_{\lambda_{1}}^{-1}
$$

Proof. The argument is by implicit induction on the last box to be added to the Ferrers diagram of $\lambda$. Suppose $\lambda_{i}=0$ for $i>k$ and $\lambda_{k} \geq 1$. Define $\lambda^{\prime}$ by $\lambda_{i}^{\prime}=\lambda_{i}$ for all $i$ except $\lambda_{k}^{\prime \prime}=\lambda_{k}-1$. Denote the product on the left side of (35) by $A(\lambda)$, then

$$
\begin{aligned}
\frac{A(\lambda)}{A\left(\lambda^{\prime}\right)} & =\prod_{i=1}^{k-1} \frac{1-q^{\lambda_{i}-\lambda_{k}+1} t^{k-i+1}}{1-q^{\lambda_{i}-\lambda_{k}+1} t^{k-i}} \prod_{j=k+1}^{L} \frac{1-q^{\lambda_{k}} j^{j-k}}{1-q^{\lambda_{k}} t^{i-k+1}} \\
& =\frac{1-q^{\lambda_{k} t}}{1-q^{\lambda_{k}} t^{L-k+1}} \prod_{i=1}^{k-1} \frac{1-q^{\lambda_{i}-\lambda_{k}+1} t^{k-i+1}}{1-q^{\lambda_{i}-\lambda_{k}+1} t^{k-i}}
\end{aligned}
$$

the $j$-product telescopes. Adjoining a box at $\left(k, \lambda_{k}\right)$ to the diagram of $\lambda^{\prime}$ causes these changes: $\operatorname{leg}\left(i, \lambda_{k} ; \lambda\right)=\operatorname{leg}\left(i, \lambda_{k} ; \lambda^{\prime}\right)+1$ for $1 \leq i<k, \operatorname{arm}(k, j ; \lambda)=\operatorname{arm}\left(k, j ; \lambda^{\prime}\right)+1=$ $\lambda_{k}-j$ for $1 \leq j<\lambda_{k}$. The calculation also uses $\operatorname{arm}\left(i, \lambda_{k} ; \lambda\right)=\operatorname{arm}\left(i, \lambda_{k} ; \lambda^{\prime}\right)=\lambda_{i}-\lambda_{k}$; $\operatorname{leg}\left(k, j ; \lambda^{\prime}\right)=\operatorname{leg}(k, j ; \lambda)=0$. Thus

$$
\frac{h_{q, t}(q t ; \lambda)}{h_{q, t}\left(q t ; \lambda^{\prime}\right)}=\left(1-q^{\lambda_{k}} t\right) \prod_{i=1}^{k-1} \frac{1-q^{\lambda_{i}-\lambda_{k}+1} t^{k-i+1}}{1-q^{\lambda_{i}-\lambda_{k}+1} t^{k-i}}
$$

because the change in the product for row $\# k$ is

$$
\prod_{j=1}^{\lambda_{k}}\left(1-q t q^{\lambda_{k}-j}\right) \prod_{j=1}^{\lambda_{k}-1}\left(1-q t q^{\lambda_{k}-1-j}\right)^{-1}=1-q^{\lambda_{k}} t .
$$

Denote the second product in (35) by $B(\lambda)$ then

$$
\frac{B(\lambda)}{B\left(\lambda^{\prime}\right)}=\frac{\left(q t^{L-k+1} ; q\right)_{\lambda_{k}-1}}{\left(q t^{L-k+1} ; q\right)_{\lambda_{k}}}=\frac{1}{1-q^{\lambda_{k} t^{L-k+1}}} .
$$

Hence $\frac{A(\lambda)}{A\left(\lambda^{\prime}\right)}=\frac{h_{q, t}(q t ; \lambda) B(\lambda)}{h_{q, t}\left(q t ; \lambda^{\prime}\right) B\left(\lambda^{\prime}\right)}$. To start the induction let $\lambda=(1,0, \ldots, 0)$, then $A(\lambda)=\prod_{j=2}^{L} \frac{1-q t^{j-1}}{1-q t^{j}}=\frac{1-q t}{1-q t^{L}}$, while $h_{q, t}(q t ; \lambda)=1-q t$ and $B(\lambda)=\left(1-q t^{L}\right)^{-1}$. This completes the proof.

Note that $\prod_{i=1}^{L}\left(q t^{L-i+1} ; q\right)_{\lambda_{1}}=\left(q t^{L} ; q, t\right)_{\lambda}$ (the generalized $(q, t)$-Pochhammer symbol). Setting $L=N-m-1$ in the Proposition leads to another formulation:

Theorem 4. Suppose $\lambda \in \mathcal{N}_{0}^{+}$then

$$
V^{(0)}(\lambda)=q^{\beta(\lambda)} t^{e_{0}(\lambda)} \frac{\left(q t^{N} ; q, t\right)_{\lambda}\left(q t^{N-m-1} ; q, t\right)_{\lambda}}{\left(q t^{N-1} ; q, t\right)_{\lambda} h_{q, t}(q t ; \lambda)} .
$$

The same method can be applied to $V^{(1)}(\lambda)$ by using $\Xi$ (Definition 10). 
Theorem 5. Suppose $\lambda \in \mathcal{N}_{1}^{+}$then

$$
V^{(1)}(\lambda)=q^{\beta(\lambda)} t^{e_{1}(\lambda)} \frac{\left(q t^{-N} ; q, t^{-1}\right)_{\lambda}\left(q t^{-m} ; q, t^{-1}\right)_{\lambda}}{\left(q t^{1-N} ; q, t^{-1}\right){ }_{\lambda} h_{q, 1 / t}\left(q t^{-1} ; \lambda\right)} .
$$

There is a modified definition of leg-length for arbitrary compositions $\alpha \in \mathbb{N}_{0}^{N}$ :

$$
\operatorname{leg}(i, j ; \alpha)=\#\left\{r: r>i, j \leq \alpha_{r} \leq \alpha_{i}\right\}+\#\left\{r: r<i, j \leq \alpha_{r}+1 \leq \alpha_{i}\right\} .
$$

Suppose $\alpha_{i+1}>\alpha_{i}$ then

$$
\frac{h_{q, t}\left(q t, s_{i} \alpha\right)}{h_{q, t}(q t, \alpha)}=\frac{1-q^{\alpha_{i+1}-\alpha_{i}} t^{r_{\alpha}(i)-r_{\alpha}(i+1)}}{1-t q^{\alpha_{i+1}-\alpha_{i}} t^{r_{\alpha}(i)-r_{\alpha}(i+1)}}=u_{1}\left(\frac{\zeta_{\alpha, E}(i+1)}{\zeta_{\alpha, E}(i)}\right)^{-1}
$$

from [2] [p.15,Prop. 5] (the argument relates to the box at $\left(i+1, \alpha_{i}+1\right)$ in the Ferrers diagram of $\alpha$ and the change in its leg-length) so that

$$
h_{q, t}\left(q t ; \alpha^{+}\right)=\mathcal{R}_{1}(\alpha, E)^{-1} h_{q, t}(q t ; \alpha) .
$$

Suppose $\alpha \in \mathcal{N}_{0}$ then from $V^{(0)}(\alpha)=\mathcal{R}_{1}(\alpha, E)^{-1} V^{(0)}\left(\alpha^{+}\right)$(see Proposition (3)) and (27) we obtain

$$
V^{(0)}(\alpha)=q^{\beta(\alpha)} t^{e_{0}\left(\alpha^{+}\right)} \frac{\left(q t^{N} ; q, t\right) \alpha_{\alpha^{+}}\left(q t^{N-m-1} ; q, t\right)_{\alpha^{+}}}{\left(q t^{N-1} ; q, t\right)_{\alpha^{+}} h_{q, t}\left(q t ; \alpha^{+}\right)} .
$$

There is a slight complication for type (1) $V^{(1)}(\alpha)=(-1)^{\operatorname{inv}(\alpha)} \mathcal{R}_{0}(\alpha, F)^{-1} V^{(1)}\left(\alpha^{+}\right)$

$$
V^{(1)}(\alpha)=(-t)^{-\operatorname{inv}(\alpha)} V^{(1)}\left(\alpha^{+}\right) \frac{h_{q, 1 / t}\left(q t^{-1} ; \alpha^{+}\right)}{h_{q, 1 / t}\left(q t^{-1} ; \alpha\right)} .
$$

Start with $\zeta_{\alpha, F}(i)=q^{\alpha_{i}} t^{r_{\alpha}(i)-1-m}$ for $1 \leq i \leq m$ (because $\left.c(i, F)=i-m-1\right)$ and then $\frac{\zeta_{\alpha, F}(i+1)}{\zeta_{\alpha, F}(i)}=q^{\alpha_{i+1}-\alpha_{i}} t^{r_{\alpha}(i+1)-r_{\alpha}(i)}$. Suppose $\alpha_{i+1}>\alpha_{i}$ and apply $\Xi$ in (36) to obtain

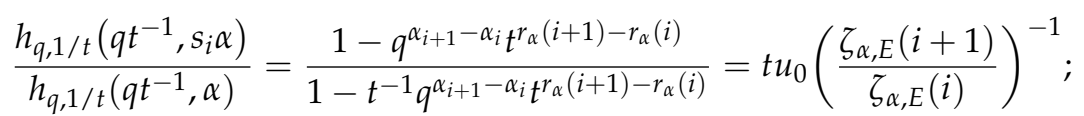

combine with $V^{(1)}\left(s_{i} \alpha\right)=-u_{0}\left(\frac{\zeta_{\alpha, E}(i+1)}{\zeta_{\alpha, E}(i)}\right) V^{(1)}(\alpha)$ and then

$$
V^{(1)}(\alpha) h_{q, 1 / t}\left(q t^{-1}, \alpha\right)=-t^{-1} V^{(1)}\left(s_{i} \alpha\right) h_{q, 1 / t}\left(q t^{-1}, s_{i} \alpha\right) .
$$

Thus, $V^{(1)}(\alpha) h_{q, 1 / t}\left(q t^{-1}, \alpha\right)=(-t)^{-\operatorname{inv}(\alpha)} V^{(1)}\left(\alpha^{+}\right) h_{q, 1 / t}\left(q t^{-1}, \alpha^{+}\right)$, and

$$
V^{(1)}(\alpha)=(-t)^{-\operatorname{inv}(\alpha)} q^{\beta(\alpha)} t^{e_{0}\left(\alpha^{+}\right)} \frac{\left(q t^{-N} ; q, t^{-1}\right)_{\alpha^{+}}\left(q t^{-m} ; q, t^{-1}\right)_{\alpha^{+}}}{\left(q t^{1-N} ; q, t^{-1}\right)_{\alpha^{+}} h_{q, 1 / t}\left(q t^{-1} ; \alpha\right)} .
$$

We have shown that the values of certain Macdonald superpolynomials at special points $\left(1, t, \ldots, t^{N-1}\right)$ or $\left(1, t^{-1}, t^{-2}, \ldots, t^{1-N}\right)$ are products of linear factors of the form $1-q^{a} t^{b}$ where $a \in \mathbb{N}$ and $-N \leq b \leq-N$.

\section{Restricted Symmetrization and Antisymmetrization}

A type of symmetric Macdonald superpolynomial has been investigated by Blondeau et al. [8]. The operators used in their work to define Macdonald polynomials are significantly different from ours. There are results on evaluations for these polynomials found by 
González and Lapointe [9]. In this section, we consider symmetrization over a subset of the coordinates, and associated evaluations.

Fix $\lambda \in \mathcal{N}_{0}^{+}$and consider the sum $p_{\lambda}^{s}=\sum\left\{c_{\beta} M_{\beta, E}: \beta^{+}=\lambda, \beta \in \mathcal{N}_{0}\right\}$ satisfying $\left(\boldsymbol{T}_{i}-t\right) p_{\lambda}^{s}=0$ for $1 \leq i<N-m-1$. In this section, we determine $p_{\lambda}^{s}\left(x^{(0)} ; \theta\right)$. Similarly fix $\lambda \in \mathcal{N}_{1}^{+}$and consider the sum $p_{\lambda}^{a}=\sum\left\{c_{\beta} M_{\beta, F}: \beta^{+}=\lambda, \beta \in \mathcal{N}_{1}\right\}$ satisfying $\left(\boldsymbol{T}_{i}+1\right) p_{\lambda}^{a}=0$ for $1 \leq i<m$, then evaluate $p_{\lambda}^{a}\left(x^{(1)} ; \theta\right)$.

Lemma 11. Suppose $\beta \in \mathbb{N}_{0}^{N}, E^{\prime} \in \mathcal{Y}_{0} \cup \mathcal{Y}_{1}$ and $\beta_{i}<\beta_{i+1}$ for some i. Let $z=\zeta_{\beta, E^{\prime}}(i+1) / \zeta_{\beta, E^{\prime}}$ (i) and let $p=c_{0} M_{s_{i} \beta, E^{\prime}}+c_{1} M_{\beta, E^{\prime}}$. If $c_{1}=\frac{t-z}{1-z} c_{0}$ then $\left(\boldsymbol{T}_{i}-t\right) p=0$ and if $c_{1}=-\frac{1-t z}{1-z} c_{0}$ then $\left(\boldsymbol{T}_{i}+1\right) p=0$.

Proof. The general transformation rules are given in matrix form with respect to the basis $\left[M_{\beta, E^{\prime}}, M_{s_{i} \beta, E^{\prime}}\right]$

$$
\boldsymbol{T}_{i}=\left[\begin{array}{cc}
-\frac{1-t}{1-z} & \frac{(1-t z)(t-z)}{(1-z)^{2}} \\
1 & \frac{z(1-t)}{1-z}
\end{array}\right] .
$$

One directly verifies that,

$$
\left(\boldsymbol{T}_{i}-t\right)\left[\begin{array}{c}
t-z \\
1-z \\
1
\end{array}\right]=\left[\begin{array}{l}
0 \\
0
\end{array}\right],\left(\boldsymbol{T}_{i}+1\right)\left[\begin{array}{c}
-\frac{1-t z}{1-z} \\
1
\end{array}\right]=\left[\begin{array}{l}
0 \\
0
\end{array}\right] .
$$

Definition 12. For $\lambda \in \mathcal{N}_{0}^{+} \operatorname{set} p_{\lambda}^{s}(x ; \theta):=\sum_{\alpha \in \mathcal{N}_{0,}, \alpha^{+}=\lambda} \mathcal{R}_{0}(\alpha, E) M_{\alpha, E}(x ; \theta)$.

Proposition 23. Suppose $\lambda \in \mathcal{N}_{0}^{+}$then $p_{\lambda}^{s}(x ; \theta)$ satisfies $\left(\boldsymbol{T}_{i}-t\right) p_{\lambda}^{\prime}=0$ for $1 \leq i<N-m-1$.

Proof. Fix $i$. If $\alpha \in \mathcal{N}_{0}, \alpha^{+}=\lambda$ and $\alpha_{i}=\alpha_{i+1}$ then $\left(\boldsymbol{T}_{i}-t\right) M_{\alpha, E}=0$ because $r_{\alpha}(i+1)=$ $r_{\alpha}(i)+1$ and thus $\zeta_{\alpha, E}(i)=t \zeta_{\alpha, E}(i+1)$. Otherwise take $\alpha_{i}<\alpha_{i+1}$ and $z=\zeta_{\alpha, E}(i+1)$ $/ \zeta_{\alpha, E}(i)$, then set

$$
p_{\alpha, i}:=\mathcal{R}_{0}\left(s_{i} \alpha, E\right) M_{s_{i} \alpha, E}+\mathcal{R}_{0}(\alpha, E) M_{\alpha, E}=\mathcal{R}_{0}\left(s_{i} \alpha, E\right)\left\{M_{s_{i} \alpha, E}+u_{0}(z) M_{\alpha, E}\right\}
$$

by Lemma 4 , and $u_{0}(z)=\frac{t-z}{1-z}$. By Lemma $11\left(\boldsymbol{T}_{i}-t\right) p_{a, i}=0$. For each $i$ the sum for $p_{\lambda}^{\prime}$ splits into singletons $\left(\alpha: \alpha_{i}=\alpha_{i+1}\right)$ and pairs $\left(\beta, s_{i} \beta: \beta_{i}<\beta_{i+1}\right)$. Each piece is annihilated by $T_{i}-t$.

There is now enough information on hand to find $p_{\lambda}^{s}\left(x^{(0)} ; \theta\right)$, since

$$
\begin{aligned}
p_{\lambda}^{s}\left(x^{(0)} ; \theta\right) & =\sum_{\alpha \in \mathcal{N}_{0, \alpha^{+}}=\lambda} \mathcal{R}_{0}(\alpha, E) M_{\alpha, E}\left(x^{(0)} ; \theta\right) \\
& =\sum_{\alpha \in \mathcal{N}_{0, \alpha^{+}}=\lambda} \frac{\mathcal{R}_{0}(\alpha, E)}{\mathcal{R}_{1}(\alpha, E)} M_{\lambda, E}\left(x^{(0)} ; \theta\right) .
\end{aligned}
$$

This sum can be evaluated using the norm formula established in [1]. This formula applies to arbitrary $\lambda \in \mathbb{N}_{0}^{N,+}$ and arbitrary sets $E^{\prime} \in \mathcal{Y}_{0} \cup \mathcal{Y}_{1}$, In the present context which 
uses only $\alpha \in \mathcal{N}_{0}$ with $\alpha^{+}=\lambda$ the formula is used with $N$ replaced by $N-m-1$ and the reverse $\lambda^{-}$of $\lambda$ is replaced by

$$
R_{0} \lambda=\left(\lambda_{N-m-1}, \ldots, \lambda_{2}, \stackrel{N-m-1}{\lambda_{1}}, 0 \ldots, 0\right) .
$$

For $n=0,1,2, \ldots$ define $[n]_{t} !=\prod_{i=1}^{n}[i]_{t}$. For $\lambda \in \mathcal{N}_{0}^{+}$and $j \leq \lambda_{1}$ let $n_{j}(\lambda)=$ $\#\left\{l: l<N-m, \lambda_{l}=j\right\}$. The formula from [1] specializes to

$$
\sum_{\alpha \in \mathcal{N}_{0}, \alpha^{+}=\lambda} \frac{\mathcal{R}_{0}(\alpha, E)}{\mathcal{R}_{1}(\alpha, E)}=\frac{[N-m-1]_{t} !}{\prod_{j=0}^{\lambda_{1}}\left[n_{j}(\lambda)\right]_{t} !} \frac{1}{\mathcal{R}_{1}\left(R_{0} \lambda, E\right)} .
$$

Note that the multiplier is a type of $t$-multinomial symbol. It is straightforward to show

$$
\mathcal{R}_{1}\left(R_{0} \lambda, E\right)=\prod_{\substack{1 \leq i<j<N-m \\ \lambda_{i}>\lambda_{j}}} \frac{1-q^{\lambda_{i}-\lambda_{j} t j-i+1}}{1-q^{\lambda_{i}-\lambda_{j} j^{j-i}}} .
$$

This product can be combined with the $(i, j)$-product in $(27)$ to show:

$$
\begin{aligned}
& p_{\lambda}^{s}\left(x^{(0)} ; \theta\right)=q^{\beta(\lambda)} t^{e_{0}(\lambda)} \frac{[N-m-1]_{t} !}{\prod_{j=0}^{\lambda_{1}}\left[n_{j}(\lambda)\right]_{t} !} \frac{\left(q t^{N} ; q, t\right)_{\lambda}}{\left(q t^{N-1} ; q, t\right)_{\lambda}} \\
& \times \prod_{\substack{1 \leq i<j<N-m \\
\lambda_{i}>\lambda_{j}}} \frac{\left(q t^{j-i+1}, q\right)_{\lambda_{i}-\lambda_{j}-1}}{\left(q t^{j-i}, q\right)_{\lambda_{i}-\lambda_{j}-1}} \tau_{E}(\theta) .
\end{aligned}
$$

Furthermore, by (3)

$$
p_{\lambda}^{s}\left(x^{(0)} ; \theta\right)=\frac{[N-m-1]_{t} !}{\prod_{j=0}^{\lambda_{1}}\left[n_{j}(\lambda)\right]_{t} !} V^{(0)}\left(R_{0} \lambda\right) \tau_{E}(\theta)
$$

Definition 13. For $y \in \mathbb{R}^{N-m-1}$ let $y^{(0)}=\left(y_{1}, \ldots, y_{N-m-1}, t^{N-m-1}, \ldots, t^{N-2}, t^{N-1}\right)$.

Lemma 3 applies to each $M_{\alpha, E}$ in the sum for $p_{\lambda}^{s}\left(y^{(0)} ; \theta\right)$ thus $\left(T_{i}-t\right) p_{\lambda}^{s}\left(y^{(0)} ; \theta\right)=0$ for $1 \leq i<N-m-1$.

Proposition 24. $p_{\lambda}^{s}\left(y^{(0)} ; \theta\right)$ is symmetric in $y$. In particular $p_{\lambda}^{s}\left(x^{(0)} u ; \theta\right)=p_{\lambda}^{s}\left(x^{(0)} ; \theta\right)$ for any permutation $u$ of $\{1,2, \ldots, N-m-1\}$ (that is $u \in \mathcal{S}_{N-m-1} \times I d_{m+1}$ ).

Proof. Suppose $1 \leq i<N-m-1$ then

$$
\begin{aligned}
& t p_{\lambda}^{s}\left(y^{(0)} ; \theta\right)=T_{i} p_{\lambda}^{s}\left(y^{(0)} ; \theta\right) \\
&=b\left(y^{(0)}, i\right) p_{\lambda}^{s}\left(y^{(0)} ; \theta\right)+\left(T_{i}-b\left(y^{(0)}, i\right)\right) p_{\lambda}^{s}\left(y^{(0)} s_{i} ; \theta\right) \\
&=b\left(y^{(0)}, i\right) p_{\lambda}^{s}\left(y^{(0)} ; \theta\right)+\left(t-b\left(y^{(0)}, i\right)\right) p_{\lambda}^{s}\left(y^{(0)} s_{i} ; \theta\right) . . \\
&\left(t-b\left(y^{(0)}, i\right)\right) p_{\lambda}^{s}\left(y^{(0)} ; \theta\right)=\left(t-b\left(y^{(0)}, i\right)\right) p_{\lambda}^{s}\left(y^{(0)} s_{i} ; \theta\right) .
\end{aligned}
$$


The latter is a polynomial identity (after multiplying by $y_{i}-y_{i+1}$ ) and thus holds for all $y^{(0)}$, and hence $p_{\lambda}^{s}\left(y^{(0)} s_{i} ; \theta\right)=p_{\lambda}^{s}\left(y^{(0)} ; \theta\right)$.

Next we consider asymmetric polynomials in type (1). Recall $\alpha \in \mathcal{N}_{1}$ implies $\zeta_{\alpha, F}(i)=$ $q^{\alpha_{i}} t^{r_{\alpha}(i)-m-1}$ if $1 \leq i \leq m$ and $\zeta_{\alpha, F}(i)=t^{N-i}$ if $i>m$.

Definition 14. For $\lambda \in \mathcal{N}_{1}^{+}$set $p_{\lambda}^{a}(x ; \theta):=\sum_{\alpha \in \mathcal{N}_{1}, \alpha^{+}=\lambda}(-1)^{\operatorname{inv}(\alpha)} \mathcal{R}_{1}(\alpha, F) M_{\alpha, F}(x ; \theta)$.

Proposition 25. Suppose $\lambda \in \mathcal{N}_{1}^{+}$then $p_{\lambda}^{a}(x ; \theta)$ satisfies $\left(\boldsymbol{T}_{i}+t\right) p_{\lambda}^{a}=0$ for $1 \leq i<m$.

Proof. Fix $i$. If $\alpha \in \mathcal{N}_{1}, \alpha^{+}=\lambda$ and $\alpha_{i}=\alpha_{i+1}$ then $\left(\boldsymbol{T}_{i}+t\right) M_{\alpha, F}=0$ because $r_{\alpha}(i+1)=$ $r_{\alpha}(i)+1$ and thus $\zeta_{\alpha, F}(i)=t^{-1} \zeta_{\alpha, F}(i+1)$. Otherwise take $\alpha_{i}<\alpha_{i+1}$ and $z=\zeta_{\alpha, E}(i+1) / \zeta_{\alpha, E}(i)$, then set

$$
\begin{aligned}
p_{\alpha, i}: & =(-1)^{\operatorname{inv}\left(s_{i} \alpha\right)} \mathcal{R}_{1}\left(s_{i} \alpha, F\right) M_{s_{i} \alpha, F}+(-1)^{\operatorname{inv}(\alpha)} \mathcal{R}_{1}(\alpha, F) M_{\alpha, F} \\
& =(-1)^{\operatorname{inv}\left(s_{i} \alpha\right)} \mathcal{R}_{1}\left(s_{i} \alpha, F\right)\left\{M_{s_{i} \alpha, F}-u_{1}(z) M_{\alpha, F}\right\}
\end{aligned}
$$

by Lemma 4 , and $u_{1}(z)=\frac{1-t z}{1-z}$. By Lemma $11\left(\boldsymbol{T}_{i}+1\right) p_{a, i}=0$. For each $i$ the sum for $p_{\lambda}^{a}$ splits into singletons $\left(\alpha: \alpha_{i}=\alpha_{i+1}\right)$ and pairs $\left(\beta, s_{i} \beta: \beta_{i}<\beta_{i+1}\right)$. Each piece is annihilated by $\boldsymbol{T}_{i}+1$.

Similarly to the symmetric case we can determine $p_{\lambda}^{a}\left(x^{(1)} ; \theta\right)$, since (by Proposition 5)

$$
\begin{aligned}
p_{\lambda}^{a}\left(x^{(1)} ; \theta\right) & =\sum_{\alpha \in \mathcal{N}_{1}, \alpha^{+}=\lambda}(-1)^{\operatorname{inv}(\alpha)} \mathcal{R}_{1}(\alpha, F) M_{\alpha, F}\left(x^{(1)} ; \theta\right) \\
& =\sum_{\alpha \in \mathcal{N}_{1}, \alpha^{+}=\lambda} \frac{\mathcal{R}_{1}(\alpha, F)}{\mathcal{R}_{0}(\alpha, F)} M_{\lambda, F}\left(x^{(1)} ; \theta\right) .
\end{aligned}
$$

Formula (37) can be adapted to find the sum by applying $\Xi$ and chasing powers of $t$ (in $\left[n_{i}(\lambda)\right]_{t}$ ! for example). The typical term in $\frac{\mathcal{R}_{1}(\alpha, F)}{\mathcal{R}_{0}(\alpha, F)}$ is

$$
\frac{1-t q^{\alpha_{j}-\alpha_{i}} t^{r_{\alpha}(j)-c_{\alpha}(i)}}{t-q^{\alpha_{j}-\alpha_{i}} t^{r_{\alpha}(j)-c_{\alpha}(i)}}=t^{-1} \frac{1-q^{\alpha_{j}-\alpha_{i}} t^{r_{\alpha}(j)-c_{\alpha}(i)+1}}{1-q^{\alpha_{j}-\alpha_{i}} t^{r_{\alpha}(j)-c_{\alpha}(i)-1}}
$$

and applying $\Xi$ yields

$$
t \frac{1-q^{\alpha_{j}-\alpha_{i}} t^{r_{\alpha}(i)-c_{\alpha}(j)-1}}{1-q^{\alpha_{j}-\alpha_{i}} t^{r_{\alpha}(i)-c_{\alpha}(j)+1}}=\frac{u_{0}(z)}{u_{1}(z)}
$$

with $z=q^{\alpha_{j}-\alpha_{i}} t^{r_{\alpha}(i)-c_{\alpha}(j)}$, the typical term in $\frac{\mathcal{R}_{0}(\alpha, E)}{\mathcal{R}_{1}(\alpha, E)}$ (after the interchange $m \longleftrightarrow N-m-1$ ). Thus

$$
\sum_{\alpha \in \mathcal{N}_{1, \alpha^{+}=\lambda}} \frac{\mathcal{R}_{1}(\alpha, F)}{\mathcal{R}_{0}(\alpha, F)}=\Xi \sum_{\alpha^{+}=\lambda} \frac{\mathcal{R}_{0}(\alpha, E)}{\mathcal{R}_{1}(\alpha, E)} .
$$


From $\Xi[n]_{t}=[n]_{1 / t}=t^{1-n}[n]_{t}$ and $n_{j}(\lambda)=\#\left\{l: l \leq m, \lambda_{l}=j\right\}$ it follows that

$$
\begin{aligned}
\frac{[m]_{1 / t} !}{\prod_{j}\left[n_{j}(\lambda)\right]_{1 / t} !} & =t^{A} \frac{[m]_{t} !}{\prod_{j=0}^{\lambda_{1}}\left[n_{j}(\lambda)\right]_{t} !}, \\
A & =-\frac{m(m-1)}{2}+\sum_{j \geq 0} \frac{n_{j}(\lambda)\left(n_{j}(\lambda)-1\right)}{2} \\
& =-\frac{1}{2} m^{2}+\frac{1}{2} m+\frac{1}{2} \sum_{j \geq 0} n_{j}(\lambda)^{2}-\frac{1}{2} m .
\end{aligned}
$$

Now let $R_{1} \lambda=\left(\lambda_{m}, \lambda_{m-1}, \ldots, \lambda_{1}, 0 \ldots, 0\right)$ and consider

$$
\mathcal{R}_{0}\left(R_{1} \lambda, F\right)=\prod_{\substack{1 \ell i<j \leq m \\
\lambda_{i} \cdot \lambda_{j}}} \frac{t-q^{\lambda_{i}-\lambda_{j}} t^{i-j}}{1-q^{\lambda_{i}-\lambda_{j} t^{i-j}}}=t^{\operatorname{inv}\left(R_{1} \lambda\right)} \prod_{\begin{array}{r}
1 \ell i<j \leq m \\
\lambda_{i} \cdot \lambda_{j}
\end{array}} \frac{1-q^{\lambda_{i}-\lambda_{j}} t^{i-j-1}}{1-q^{\lambda_{i}-\lambda_{j} t^{i-j}}}
$$

and the transformed

$$
\begin{aligned}
\Xi \mathcal{R}_{1}\left(R_{0} \lambda, E\right) & =\prod_{\substack{1 \ell i<j \leq m \\
\lambda_{i} \cdot \lambda_{j}}} \frac{1-q^{\lambda_{i}-\lambda_{j}} t^{i-j-1}}{1-q^{\lambda_{i}-\lambda_{j} t^{i-j}}} \\
& =t^{-\operatorname{inv}\left(R_{1} \lambda\right)} \mathcal{R}_{0}\left(R_{1} \lambda, F\right) .
\end{aligned}
$$

This results in

$$
\sum_{\alpha \in \mathcal{N}_{1,}, \alpha^{+}=\lambda} \frac{\mathcal{R}_{1}(\alpha, F)}{\mathcal{R}_{0}(\alpha, F)}=t^{A+\operatorname{inv}\left(R_{1} \lambda\right)} \frac{[m]_{t} !}{\prod_{j=0}^{\lambda_{1}}\left[n_{j}(\lambda)\right]_{t} !} \frac{1}{\mathcal{R}_{0}\left(R_{1} \lambda, F\right)} .
$$

We find

$$
\begin{aligned}
\operatorname{inv}\left(R_{1} \lambda\right) & =\sum_{1 \leq i<j \leq \lambda_{1}} n_{i}(\lambda) n_{j}(\lambda)=\frac{1}{2}\left\{\left(\sum_{i=1}^{\lambda_{1}} n_{i}(\lambda)\right)^{2}-\sum_{i=1}^{\lambda_{1}} n_{i}(\lambda)^{2}\right\} \\
& =\frac{1}{2} m^{2}-\frac{1}{2} \sum_{i=1}^{\lambda_{1}} n_{i}(\lambda)^{2}=-A,
\end{aligned}
$$

and we have shown

$$
\begin{aligned}
p_{\lambda}^{a}\left(x^{(1)} ; \theta\right) & =\frac{[m]_{t} !}{\prod_{j=0}^{\lambda_{1}}\left[n_{j}(\lambda)\right]_{t} !} \frac{1}{\mathcal{R}_{0}\left(R_{1} \lambda, F\right)} M_{\lambda, F}\left(x^{(1)} ; \theta\right) \\
& =(-1)^{\operatorname{inv}\left(R_{1} \lambda\right)}[m]_{t} !\left\{\prod_{j=0}^{\lambda_{1}}\left[n_{j}(\lambda)\right]_{t} !\right\}^{-1} V^{(1)}\left(R_{1} \lambda\right) \tau_{F}(\theta)
\end{aligned}
$$

Similarly to type (0) this formula can be further developed:

$$
\mathcal{R}_{0}\left(R_{1} \lambda, F\right)^{-1}=\prod_{\substack{1 \leq i<j \leq m \\ \lambda_{i}>\lambda_{j}}} \frac{1-q^{\lambda_{i}-\lambda_{j}} t^{i-j}}{t-q^{\lambda_{i}-\lambda_{j}} t^{i-j}}=t^{-\operatorname{inv}\left(R_{1} \lambda\right)} \prod_{\substack{1 \leq i<j \leq m \\ \lambda_{i}>\lambda_{j}}} \frac{1-q^{\lambda_{i}-\lambda_{j}} t^{i-j}}{1-q^{\lambda_{i}-\lambda_{j}} t^{i-j-1}}
$$


Thus, (from Theorem 3)

$$
\begin{aligned}
p_{\lambda}^{a}\left(x^{(1)} ; \theta\right) & =q^{\beta(\lambda)} t^{A(\lambda)} \frac{[m]_{t} !}{\prod_{j=0}^{\lambda_{1}}\left[n_{j}(\lambda)\right]_{t} !} \frac{\left(q t^{-N} ; q, t^{-1}\right)_{\lambda}}{\left(q t^{1-N} ; q, t^{-1}\right)_{\lambda}} \\
& \times \prod_{\substack{1 \leq i<j<N-m \\
\lambda_{i}>\lambda_{j}}} \frac{\left(q t^{i-j-1}, q\right)_{\lambda_{i}-\lambda_{j}-1}}{\left(q t^{i-j}, q\right)_{\lambda_{i}-\lambda_{j}-1}} \tau_{F}, \\
A(\lambda) & =\sum_{i=1}^{m} \lambda_{i}(N-m-i)-\operatorname{inv}\left(R_{1} \lambda\right), \\
\operatorname{inv}\left(R_{1} \lambda\right) & =\frac{1}{2} m^{2}-\frac{1}{2} \sum_{i=1}^{\lambda_{1}} n_{i}(\lambda)^{2} .
\end{aligned}
$$

Definition 15. For $y \in \mathbb{R}^{m}$ let $y^{(1)}=\left(y_{1}, \ldots, y_{m}, t^{-m}, \ldots, t^{2-N}, t^{1-N}\right)$

Lemma 5 applies to each $M_{\alpha, F}$ in the sum for $p_{\lambda}^{a}\left(y^{(1)} ; \theta\right)$ thus $\left(T_{i}+1\right) p_{\lambda}^{a}\left(y^{(1)} ; \theta\right)=0$ for $1 \leq i<m$.

Proposition 26. $p_{\lambda}^{a}\left(y^{(1)} ; \theta\right)$ is symmetric in $y$. In particular $p_{\lambda}^{a}\left(x^{(1)} u ; \theta\right)=p_{\lambda}^{a}\left(x^{(1)} ; \theta\right)$ for any permutation $u$ of $\{1,2, \ldots, m\}$ (that is $u \in \mathcal{S}_{m} \times I d_{N-m}$ ).

Proof. Suppose $1 \leq i<m$ then

$$
\begin{aligned}
-p_{\lambda}^{a}\left(y^{(1)} ; \theta\right) & =\boldsymbol{T}_{i} p_{\lambda}^{a}\left(y^{(1)} ; \theta\right) \\
& =b\left(y^{(1)}, i\right) p_{\lambda}^{s a}\left(y^{(1)} ; \theta\right)+\left(T_{i}-b\left(y^{(1)}, i\right)\right) p_{\lambda}^{a}\left(y^{(1)} s_{i} ; \theta\right) \\
& =b\left(y^{(1)}, i\right) p_{\lambda}^{a}\left(y^{(1)} ; \theta\right)-\left(1+b\left(y^{(1)}, i\right)\right) p_{\lambda}^{a}\left(y^{(1)} s_{i} ; \theta\right) \\
\left(1+b\left(y^{(1)}, i\right)\right) p_{\lambda}^{a}\left(y^{(1)} ; \theta\right) & =\left(1+b\left(y^{(1)}, i\right)\right) p_{\lambda}^{a}\left(y^{(1)} s_{i} ; \theta\right) .
\end{aligned}
$$

The latter is a polynomial identity (after multiplying by $y_{i}-y_{i+1}$ ) and thus holds for all $y^{(1)}$, and hence $p_{\lambda}^{a s}\left(y^{(1)} s_{i} ; \theta\right)=p_{\lambda}^{a}\left(y^{(1)} s_{i} ; \theta\right)$.

\section{Conclusions and Future Directions}

In the context of Macdonald polynomials, "evaluation" refers to finding a closed form consisting of a product of linear factors for the value of a polynomial at a certain point. The polynomials are sums of monomials whose coefficients are rational functions of $q, t$. The linear factors are of the form $1-q^{a} t^{b}$ where $a, b \in \mathbb{Z}$ and $|b| \leq N$. Any ordinary (scalar-valued) nonsymmetric Macdonald polynomial does have an evaluation formula at the point $\left(1, t, t^{2}, \ldots\right)$ (see [7] [Prop. 5]) (this is a multi-variable analog of the value of a Gegenbauer polynomial $C_{n}^{\alpha}(x)$ at $\left.x=1\right)$. However, in the vector-valued case, computational experiments suggest that there are no generally applicable formulas of this type. In the present paper we established evaluations of a relatively restricted class of nonsymmetric polynomials at special points. The labels $E^{\prime}$ of the Macdonald polynomials $M_{\alpha, E^{\prime}}$ have only two possibilities out of many, $\left(\begin{array}{c}N-1 \\ m\end{array}\right)$ for the isotype $\left(N-m, 1^{m}\right)$.

There are other possible evaluations that deserve to be investigated: these relate to singular polynomials. This refers to the situation where the parameters $q, t$ satisfy a relation like $q^{a} t^{b}=1$ and a polynomial $M_{\alpha, E^{\prime}}$ satisfies $\xi_{i} M_{\alpha, E^{\prime}}=\omega_{i} M_{\alpha, E^{\prime}}$ for $1 \leq i \leq N$ The JucysMurphy operators on $s \mathcal{P}_{m}$ are defined in terms of $\left\{\boldsymbol{T}_{i}\right\}$ (see (5)): $\boldsymbol{\omega}_{N}=1, \boldsymbol{\omega}_{i}=t^{-1} \boldsymbol{T}_{i} \boldsymbol{\omega}_{i+1} \boldsymbol{T}_{i}$ for $1 \leq i<N$. Of course, finding these singular parameters $(q, t)$ is already a research 
problem by itself. For small $N$ and degree we can find some examples (with computer algebra) and test evaluations. It appears there are interesting results to find.

Consider $N=6$ and $\mathcal{P}_{1,0}, \alpha=(1,1,0,0,0,0)$ (of isotype $(5,1)$ ). The spectral vector of $M_{\alpha,\{5,6\}}$ is $\left(q t^{4}, q t^{3}, t^{2}, t, t^{=1}, 1\right)$. Let

$$
x=\left(x_{1}, x_{2}, t^{2}, t, t^{-1}, 1\right) .
$$

The polynomial $M_{\alpha,\{5,6\}}$ is singular for $q t^{3}=1$ and

$$
\begin{aligned}
M_{\alpha,\{5,6\}}(x ; \theta) & =t^{16}\left(x_{1}-1\right)\left(x_{2}-1\right)\left(t^{4} \theta_{6}-t^{5} \theta_{5}\right), \\
\tau_{\{5,6\}} & =t^{4} \theta_{6}-t^{5} \theta_{5} .
\end{aligned}
$$

Similarly $M_{\alpha,\{4,6\}}$ is singular at $q t^{3}=1$; its spectral vector is $\left(q t^{4}, q t^{3}, t^{2}, t^{-1}, t, 1\right)$ and for $x^{\prime}=\left(x_{1}, x_{2}, t^{2}, t^{-1}, t, 1\right)$

$$
\begin{aligned}
M_{\alpha,\{4,6\}}\left(x^{\prime} ; \theta\right) & =t^{16}\left(x_{1}-1\right)\left(x_{2}-1\right) \tau_{\{4,6\}}(\theta), \\
\tau_{\{4,6\}} & =-t^{6} \theta_{4}+\frac{t^{5}}{1+t}\left(\theta_{5}+\theta_{6}\right) .
\end{aligned}
$$

As well $M_{\alpha,\{3,6\}}$ is singular at $q t^{3}=1$; its spectral vector is $\left(q t^{4}, q t^{3}, t^{-1}, t^{2}, t, 1\right)$ and for $x^{\prime \prime}=\left(x_{1}, x_{2}, t^{-1}, t^{2}, t, 1\right)$

$$
\begin{aligned}
M_{\alpha,\{3,6\}}\left(x^{\prime \prime} ; \theta\right) & =t^{16}\left(x_{1}-1\right)\left(x_{2}-1\right) \tau_{\{3,6\}}(\theta), \\
\tau_{\{3,6\}} & =-t^{7} \theta_{3}+\frac{t^{6}}{1+t+t^{2}}\left(\theta_{4}+\theta_{5}+\theta_{6}\right) .
\end{aligned}
$$

For an example with higher degree consider $N=6, \mathcal{P}_{4,1}$ and $\beta=(2,1,0,0,0,0)$, $F=\{1,2,3\}$ (the isotype is $\left.\left(3,1^{3}\right)\right)$ Then $\zeta_{\beta, F}=\left(q^{2} t^{-3}, q t^{-2}, t^{-1}, t^{2}, t, 1\right)$ and $M_{\beta, F}$ is singular for $q=t^{2}$. At $x=\left(x_{1}, x_{2}, t^{-1}, t^{2}, t, 1\right)$ we find

$$
\begin{aligned}
M_{\beta, F}(x ; \theta) & =t^{8}\left(x_{1}-t x_{2}\right)\left(x_{1}-1\right)\left(x_{2}-1\right) \tau_{F}, \\
\tau_{F} & =-\theta_{1} \theta_{2} \theta_{3}\left(\theta_{4}+\theta_{5}+\theta_{6}\right) .
\end{aligned}
$$

We would expect an evaluation formula involving the elements of the spectral vector for which $\alpha_{i}=0$ and with as many free variables as nonzero elements of $\alpha$. There is a nice necessary condition for a singular value: the $t$-exponents of the specialized spectral vector have to agree with the content vector of an RSYT. For example, set $q=t^{2}$ in $\zeta_{\beta, F}$ with the result $\left(t, 1, t^{-1}, t^{2}, t, 1\right)$, and $[1,0,-1,2,1,0]$ is the content vector of

$$
\left[\begin{array}{lll}
6 & 5 & 4 \\
3 & 2 & 1
\end{array}\right]
$$

Then $M_{\gamma, F}$ with $\gamma=(2,1,0,0,0,0)$ can not be singular at $q=t^{2}$ : the spectral vector $\zeta_{\gamma, F}=\left(1, t, t^{-1}, t^{2}, t, 1\right)$ and $[0,1,-1,2,1,0]$ is not the content vector of any RSYT.

There are interesting results dealing with singular Macdonald superpolynomials waiting to be found.

Funding: This research received no external funding.

Institutional Review Board Statement: Not applicable.

Informed Consent Statement: Not applicable.

Data Availability Statement: Not applicable. 
Conflicts of Interest: The author declares no conflict of interest.

\section{References}

1. Dunkl, C.F. Nonsymmetric Macdonald superpolynomials. arXiv 2020, arXiv:2011.05886.

2. Dunkl, C.F.; Luque, J.-G. Vector valued Macdonald polynomials. Sém. Lothar. Combin. 2012, B66b, 68.

3. Baker, T.H.; Forrester, P.J. Symmetric Jack polynomials from non-symmetric theory. Ann. Comb. 1999, 3, 159-170. [CrossRef]

4. Dipper, R.; James, G. Representations of Hecke algebras of general linear groups. Proc. Lond. Math. Soc. 1986, 52, 2-52. [CrossRef]

5. Baker, T.H.; Forrester, P.J. A q-analogue of the type A Dunkl operator and integral kernel. Int. Math. Res. Notices 1997, 14, 667-686. [CrossRef]

6. Braverman, A.; Etingof, P.; Finkelberg, M. Cyclotomic double affine Hecke algebras. Ann. Sci. L'Ecole Norm. Super. 2020, 53, 1249-1314. [CrossRef]

7. Dunkl, C.F.; Luque, J.-G. Clustering properties of rectangular Macdonald polynomials. Ann. Inst. Henri Poincaré Comb. Phys. Interact. 2015, 2, 263-307. [CrossRef]

8. Blondeau-Fournier, O.; Desrosiers, P.; Lapointe, L.; Mathieu, P. Macdonald polynomials in superspace as eigenfunctions of commuting operators. J. Combin. 2012, 3, 495-561. [CrossRef]

9. González, C.; Lapointe, L. The norm and the evaluation of the Macdonald polynomials in superspace. Eur. J. Combin. 2020, 83, 103018. [CrossRef] 